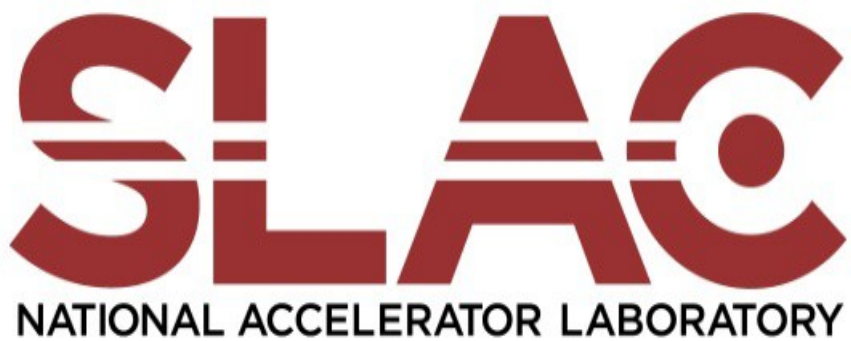

\title{
LABORATORY DIRECTED RESEARCH AND DEVELOPMENT PROGRAM
}

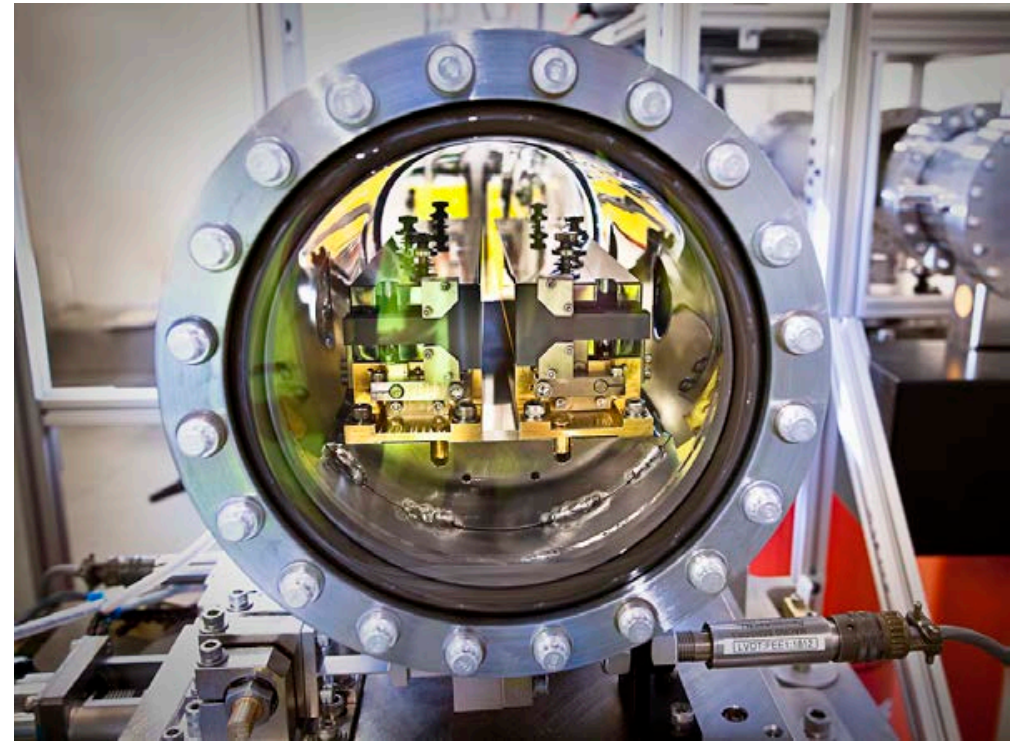

\section{FY2014 ANNUAL REPORT}

MARCH 2015 
(This page intentionally left blank) 


\section{APPROVALS}

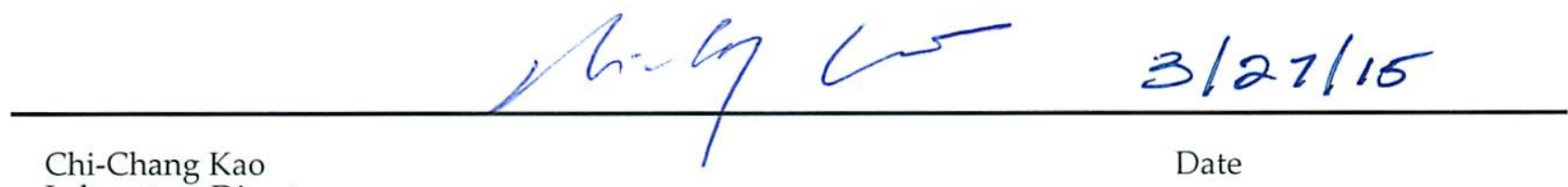

Laboratory Director

SLAC National Accelerator Laboratory

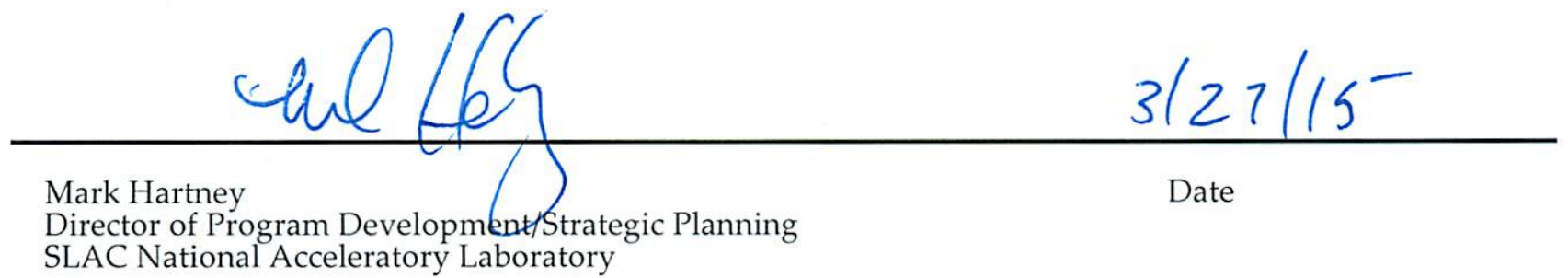

Published By:

SLAC National Accelerator Laboratory

2575 Sand Hill Road

Menlo Park, CA 94025

This document and the material and data contained herein were developed under the sponsorship of the United States Government. Neither the United States nor the Department of Energy, nor the Leland Stanford Junior University, nor their employees, makes any warranty, express or implied, or assumes any liability or responsibility for accuracy, completeness or usefulness of any information, apparatus, product or process disclosed, or represents that its use will not infringe privately owned rights. Mention of any product, its manufacturers, or suppliers shall not, nor is it intended to imply approval, disapproval, or fitness for any particular use. A royalty-free, non-exclusive right to use and disseminate it for any purpose whatsoever is expressly reserved to the United States and the University.

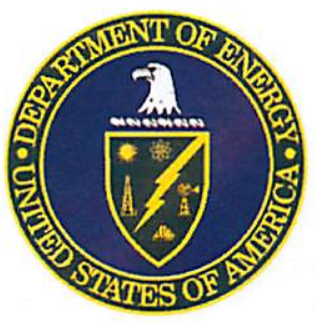




\section{Contents}

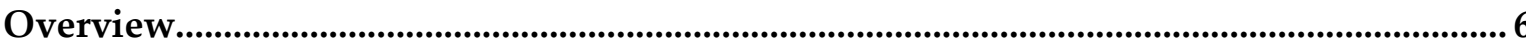

1.0 Functional Polymers for Lithium-ion Batteries...........................................................................

2.0 Spatial and time resolving pixel detector - Tixel....................................................................... 10

3.0 Understanding Electrochemically-Active Oxide Surfaces Far From Equilibrium At Elevated Temperatures.................................................................................................................... 12

4.0 Chemistry in motion: new approaches to probe enzymatic reaction mechanisms in

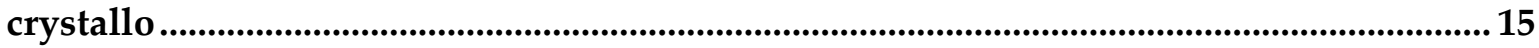

5.0 Center for Laboratory Astrophysics ........................................................................................ 18

6.0 New Initiative for Synchrotron Radiation-based Catalysis and Energy Research............ 21

7.0 Interfacial photoelectrochemistry using oxide heterostructures ............................................ 23

8.0 Project Title: Towards in-Situ Growth and Spectroscopy of Complex Oxide Thin Films Heterostructures .............................................................................................................................. 25

9.0 KIPAC Inflation Initiative (CMB) ....................................................................................... 27

10.0 Exploring the Scientific Capability of Momentum-Resolved Resonant Inelastic Soft X-ray Scattering for Material Science Research ..................................................................................... 29

11.0 Low Dimensional Materials for Energy Applications .............................................................. 32

12.0 Screening of Alloy Catalysts for Electrochemical $\mathrm{CO}_{2}$ Reduction............................................34

13.0 Correlated electron physics at oxide interfaces........................................................................... 41

14.0 Non Fermi Liquids................................................................................................................ 43

15.0 Prototype for a Microjoule-class Femtosecond XUV Source............................................... 46

16.0 Towards the SLAC Femtosecond Macromolecular Micro and Nanocrystallography Facilities

17.0 Modeling Conformational Ensembles of Proteins and Complexes .......................................54

18.0 Methodology Developments for Structural Studies of Post-translational Modifications of Proteins 58 
19.0 An Automated Pipeline for Cosmological Mock Sky Survey Production......................... 62

$20.0 \mathrm{cPix}^{2}$ : multi-gate area detector for $\mathrm{MHz}$ repetition rate pump-probe experiments .......... 65 


\section{Overview}

The Department of Energy (DOE) and the SLAC National Accelerator Laboratory (SLAC) encourage innovation, creativity, originality and quality to maintain the Laboratory's research activities and staff at the forefront of science and technology. To further advance the Laboratory's scientific research capabilities, the Laboratory allocates a portion of its funds for the Laboratory Directed Research and Development (LDRD) program. With DOE guidance, the LDRD program enables SLAC scientists to make rapid and significant contributions to seeding new strategies for solving important national science and technology problems. The LDRD program is conducted using existing research facilities. The project proposals can be characterized as:

- Small-scale research and development activities or pilot projects;

- Bench-scale research projects;

- Information gathering, computer modeling, conceptual design and feasibility studies.

In addition to building new core competencies that support the DOE missions, the LDRD project proposals may also conduct scientific research and development that support the missions of other federal agencies and/or non-federal sponsors.

The LDRD program supports the SLAC mission in several ways. First, because LDRD funds can be allocated within a relatively short time frame, SLAC researchers can support the mission of the Department of Energy (DOE) and serve the needs of the nation by quickly responding to forefront scientific problems and capitalizing on new opportunities. Second, LDRD enables SLAC to attract and retain highly qualified scientists and to support their efforts to carry out world leading research. Finally, the LDRD program also supports new projects that involve graduate students and postdoctoral fellows, thus contributing to the education mission of SLAC.

In FY2014, SLAC continued to see some of the previously funded LDRD programs transition to follow on funding with the Office of Science, (including an Early Career Award), EERE and other agencies such as DARPA and NIH. This external validation shows that the projects selected were important to continuing forefront science and use-inspired applications with mission relevance and demonstrates they were delivering significant results.

SLAC has a formal process for allocating funds for the LDRD program. The process relies on individual scientific investigators and the scientific leadership of the laboratory to identify opportunities that will contribute to scientific and institutional goals. The process is also designed to maintain compliance with DOE Orders, in particular DOE Order 413.2B (dated April 19, 2006). From year-to-year, the distribution of funds among the scientific program areas changes. This flexibility optimizes SLAC's ability to respond to emerging opportunities. SLAC LDRD policy and program decisions are the responsibility of the Laboratory Director. The Director directs the LDRD Program Manager to initiate the program each year in February and 
schedule the supporting activities. Successful proposals are usually announced in September with funds available at the beginning of the fiscal year. Normally some funds are held as unallocated to allow new ideas to be funded later in the year. After the call for proposals, the scientists confer with the scientific program ALDs before submitting their proposals. Later in the spring, review teams of distinguished researchers from inside and outside the lab meet with the proponents to hear the proposals and ask questions that may have come from reading the written proposals. The outcome of the review is a ranking of the proposals and comments back to the proposers on merits of the proposals. In the summer, the Associate Laboratory Directors meet over several weeks to review the committee's assessment, prioritize based on the lab's strategic plan, and then forward their recommendations to the Director who makes the final decisions. The list of projects is sent to the SLAC DOE Site Office for concurrence in September.

LDRD accounting procedures and financial management are consistent with the Laboratory's accounting principles and stipulations under the contract between the SLAC and the Department of Energy, with accounting maintained through the Laboratory's Chief Financial Officer and Budget Office.

In FY2014, SLAC was authorized by DOE to establish a funding ceiling for the LDRD program of $\$ 5.5 \mathrm{M}$, including General \& Administrative (G\&A) overhead. Scientists submitted 41 proposals, requesting about $\$ 9.1 \mathrm{M}$ in funding. Twenty projects were funded totaling $\$ 4.23 \mathrm{M}$, with awards ranging from $\$ 50 \mathrm{~K}$ to $\$ 500 \mathrm{~K}$.

Actual costs for the FY2014 program were \$4.4M, which equals 1.03\% of SLAC's FY2014 operating and capital equipment budget of \$331M.

Annual reports for the FY14 project activities follow. 


\subsection{Functional Polymers for Lithium-ion Batteries}

Principal Investigator: Zhenan Bao Collaborators: Yi Cui, Michael Toney.

\section{Project Description}

This project continues to develop new strategic directions within SLAC that will synergize the expertise from several fronts including polymer chemistry, nanostructure synthesis and electrochemical device testing, and structural analysis and characterization. The previous LDRDfunded projects has led to successful demonstration of the capability of making 3-D conductive polymer hydrogels coating and self-healing polymers coating for high performance lithium ion batteries anode materials (e.g., silicon). Taking the advantage of the synergistic combination of polymeric chemistry and nanomaterials, the project aims to major advancements of battery storage performance based on: 1) a continuous development of a general synthetic platform to make a class of high-performance electrode materials and 2) new hybrid electrode structure design and fundamental understanding of self-healing materials in battery operation.

\section{Accomplishments}

Major advancements were made by the continuous support of the LDRD project this year. First, we developed a simple yet efficient aqueous solution process to fabricate a class of highperformance flexible electrodes using materials comprised of interpenetrating networks of CNT and conducting polymer hydrogel. The electrodes were made from a homogenous precursor solution containing water-stable conducting polymer, e.g., poly (three, 4-ethylenedioxythiophene) doped with poly (4-styrenesulfonate) (or PEDOT: PSS), carbon nanotubes (CNT) and electrochemically active nanoparticles. Unlike previously reported conducting polymers (e.g., polyaniline, polypyrrole, polythiophene), which are mechanically fragile and incompatible with aqueous solution processing, this interpenetrating network of the CNT-conducting polymer hydrogel exhibited good mechanical properties, high conductivity, and facile ion transport, leading to facile electrode kinetics and high strain tolerance during electrode volume change. A high-rate capability for $\mathrm{TiO}_{2}$ and high cycling stability for SiNP electrodes were achieved. Typically, the flexible $\mathrm{TiO}_{2}$ electrodes achieved a capacity of $76 \mathrm{mAh} \mathrm{g}^{-1}$ in $40 \mathrm{~s}$ of charge/discharge and a high areal capacity of $2.2 \mathrm{mAh} \mathrm{cm}^{-2}$ can be obtained for flexible SiNPbased electrodes at $0.1 \mathrm{C}$ rate. Considering the vast library of conducting polymers and active nanoparticles reported so far, this simple yet efficient solution process is promising for the fabrication of a variety of high performance flexible electrodes. This work has been published in Advanced Energy Materials.

Second, we demonstrated an effective electrode design approach towards low-cost Si electrodes with long cycling life and high mass loading using self-healing polymer (SHP). Silicon (Si) holds great promise for high-capacity lithium storage, but its poor cycling stability related to structure degradation remains to be solved. Common strategies to improve cycling stability are to fabricate nanostructured electrodes with $\mathrm{Si}$ particle or domain size down to $150 \mathrm{~nm}$ or less, but their 
scalable synthesis remains highly challenging. Instead of relying on high-cost Si nanostructures, we recently showed that SHP binder could improve the cycling performance of low cost Si microparticle. However, high areal capacity with long cycling life has not been achieved. We designed a new electrode architecture with three-dimensional (3D) spatial distribution of SHP into $\mathrm{Si}$ electrodes that can promote more effective self-healing. Through systematically understanding of the Si particle size effects in the 3D Si-SHP electrodes, we discovered that large size particles (500 nm-1.5 $\mu \mathrm{m}$ in diameter) can be cycled very stability even though they fracture during cycling due to their size above the critical breaking size $(\sim 150 \mathrm{~nm})$. The effective structure enabled stable Si electrodes with high areal capacities of 3 to $4 \mathrm{mAh} \mathrm{cm}^{-2}$ for such low cost large Si particles, which can be easily produced by the existing mechanical milling process. This is critically important since high areal-capacity loading above $3 \mathrm{mAh} \mathrm{cm}^{-2}$ is needed for practical applications, but this was previously demonstrated with only a few sophisticated Si nanostructures. This simple yet efficient strategy towards low-cost fabrication of stable high mass loading Si electrodes possesses obvious advantages over previous works in the field. This work has been recently submitted for publication.

\section{Publications}

Z. Chen, J. W. F. To, C. Wang, Z. Lu, N. Liu, A. Chortos, L. Pan, F. Wei, Y. Cui, and Z. Bao, “A Three-Dimensionally Interconnected Carbon Nanotube-Conducting Polymer Hydrogel Network for HighPerformance Flexible Battery Electrodes" Adv. Energy Mater., 2014, 4. 1400207. doi:

10.1002/aenm.201400207.

Z. Chen, C. Wang, J. Lopez, Z. Lu, Y. Cui and Z. Bao, "High-Areal-Capacity Silicon Electrodes with Low-Cost Silicon Particles Based on Spatial Control of Self-Healing Binder" 2014, Submitted.

\section{Workshops}

None 


\section{Questionnaire}

\begin{tabular}{|ll|}
\hline Question & Answer \\
\hline Will follow-on funding (post-LDRD project) be applied for? & Yes \\
\hline Source of support for follow-on funding? & Stanford Global \\
& Climate and Energy \\
\hline Has follow-on funding been obtained? & Program \\
\hline Amount of follow-on funding (\$K)? & Approved \\
& \$1.699M total for \\
& three years (PI: \\
& Zhenan Bao, Co-PIs: \\
Number of Post Docs supported by LDRD project? & Yi Cui, Michael \\
\hline Number of students supported by LDRD project? & Toney) \\
\hline Number of scientific staff/technical staff hired with LDRD funding? & 1 \\
\hline Number of copyrights filed (beyond publications)? & 0 \\
\hline Number of invention disclosures filed? & 0 \\
\hline Number of patent applications filed? & 0 \\
\hline
\end{tabular}




\subsection{Spatial and time resolving pixel detector - Tixel}

Principal Investigator: Christoph Bostedt

\section{Project Description}

Spectroscopy applications for ultra-intense light sources such as the Linac Coherent Light Source free-electron laser require a new generation of time-resolving, multi-hit capable detectors. We are developing a time-resolving pixelated particle detector (Tixel) capable of detecting one hit per pixel per frame. We will demonstrate its functionality with an ultrafast laser source and subsequently develop the system into an electron and ion spectrometer.

The detector is based on silicon technology. A thin silicon pixel sensor will be coupled with fast readout electronics. Each pixel will be an independent detector channel making this detector fully multi-hit capable.

\section{Accomplishments}

During the first year of the program, we have focused our attention on developing the custom Application Specific Integrated Circuit (ASIC) and the silicon Pixel Array Detector (PAD) which will be bump bonded together to form the detector.

For the ASIC the complete architecture has been finalized and validated through extensive simulations to meet the required specifications. The ASIC is composed of three sections (c.f. figure 1): an array of pixels that perform the time measurement operation, a row selector that addresses rows of the matrix for column parallel readout, and a common section ("balcony") that is used to configure as well as control the phases of operation and readout the matrix. The design and simulations of the pixel circuitry have been completed together with parts of the layout. The timing information is extracted from the sensor signal with a charge-sensitive amplifier (CSA) followed by a discriminator. The time-to-digital conversion is performed in a two-step time measurement. The row selection logic has been designed, simulated and laid out. The

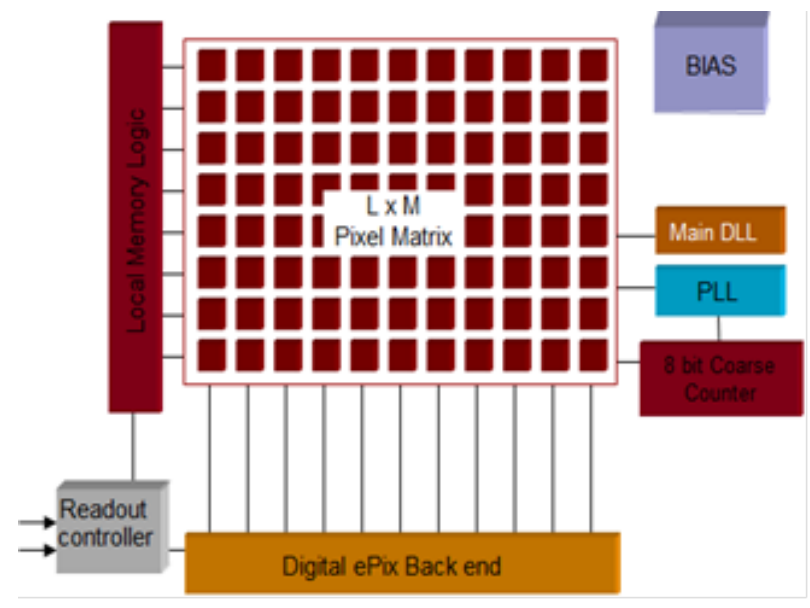

Figure 1: ASIC architecture for the tixel sensor "balcony" components have been design and simulated. In particular, the high-speed serializers have been demonstrated to sustain data rates up to $1 \mathrm{~Gb} / \mathrm{s}$ and they are compatible with the SLAC custom designed encoder based on the $\mathrm{8b} / 10 \mathrm{~b}$ protocol. The assembly of the full chip layout is now close to completion with a submission 
for production scheduled towards the end of 2014. The

submission date is slightly delayed with respect to the original schedule due to unforeseen design complications.

For the PAD, device and process simulations have been performed to design and optimize the silicon sensor. We have settled on a 50 micrometer sensor thickness that will meet or beat the $1 \mathrm{~ns}$ readout specification. For the sensor an "active edge" design is used to minimize the inactive region at the edge for optimized tiling of the Tixel detector. The mask set design has been completed. The 7-mask process flow has been defined and SLAC researchers are currently processing the sensor at the Stanford Nanofabrication facility (figure 2). The sensor process is about $80 \%$ complete at the end of the first year. Concurrently we are developing the bump-bonding process suitable for the thin (50 micron) sensor.

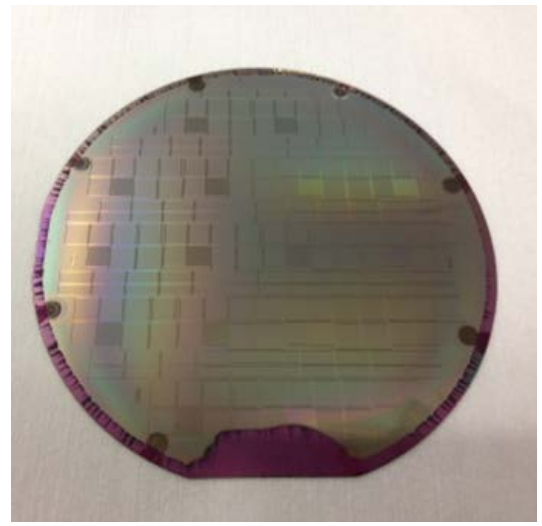

Figure 2: Silicon wafer with the tixel $P A D$ during processing at the Stanford Nanofabrication facility

\section{Publications}

None

\section{Workshops}

None

\section{Questionnaire}

Will follow-on funding (post-LDRD project) be applied for?

Planned

Source of support for follow-on funding?

TBD

Has follow-on funding been obtained?

N/A

Amount of follow-on funding $(\$ \mathrm{~K})$ ?

N/A

Number of Post Docs supported by LDRD project?

0

Number of students supported by LDRD project?

0

Number of scientific staff/technical staff hired with LDRD funding? 0

Number of copyrights filed (beyond publications)?

0

Number of invention disclosures filed? 0

Number of patent applications filed? 


\subsection{Understanding Electrochemically-Active Oxide Surfaces Far From Equilibrium At Elevated Temperatures}

Principal Investigator: W. C. Chueh

\section{Project Description}

The goal of this project is to answer the most basic question on the reactivity of oxides: how charge-transfer reactions couple to surface chemistry and structure under large driving forces. We will develop the following in-situ synchrotron-based electronic and structural analysis tools to probe electrochemically-active oxides at elevated-temperature and near ambient gas pressure: Xray photoelectron spectroscopy, absorption spectroscopy, and surface diffraction. In particular, we will investigate the reactivity of oxygen gas molecules with model perovskite oxides, which are chemically amenable to a wide range of compositions. Therefore, they are ideally suited for understanding the coupling between physical, chemical and electronic structures at electrochemical interfaces.

\section{Accomplishments}

In Year 2 of this LDRD project, we focused on developing in-situ surface X-ray diffraction (SXRD) to probe the surface atomic structure of electrochemically-active oxides under environmental conditions relevant to electrochemistry. Three atomically-flat oxide surfaces were studied: cubic zirconium oxide (fluorite), cerium oxide (fluorite), and strontium titanate (perovskite) (Figure 1). By varying temperature and oxygen pressure, we directly controlled the oxidation state of the surface, and probed their role in surface electrochemical reactions such as oxygen-reduction reactions.

A key experimental capability commissioned this year is the in-situ diffraction cell. Shown in Figure 2, this environmental cell enables precise atmosphere and polarization control for temperatures up to $1,100{ }^{\circ} \mathrm{C}$. We successfully interfaced the diffraction cell to the diffractometer at Stanford Synchrotron Radiation Lightsource beam line 7-2. By combining the cell with areal Xray detectors, SXRD patterns of single crystals were collected under in -situ conditions. We successfully carried out more than 200 hours of SXRD measurements under a wide range of 12

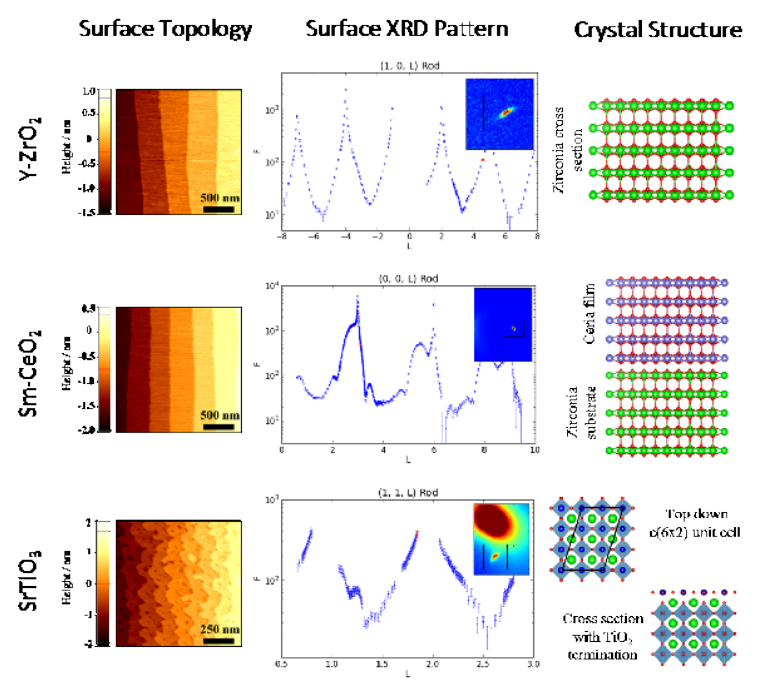

Figure 1: Atomically-flat oxide surfaces investigated in this project.

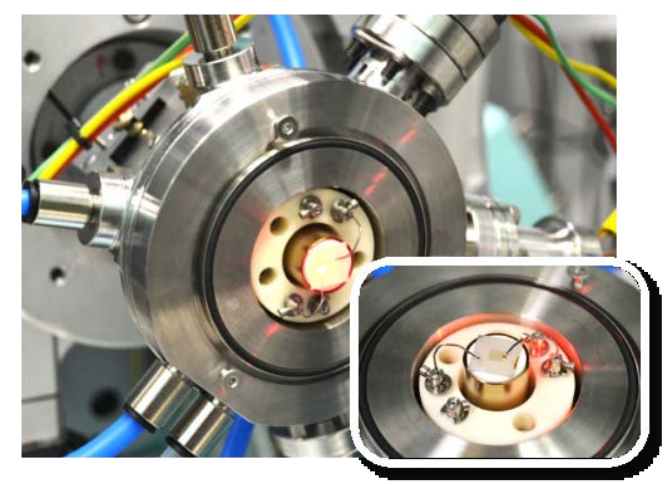

Figure 2: In-situ diffraction cell designed and fabricated in this project.

LDRD 2014 ANNUAL REPORT 


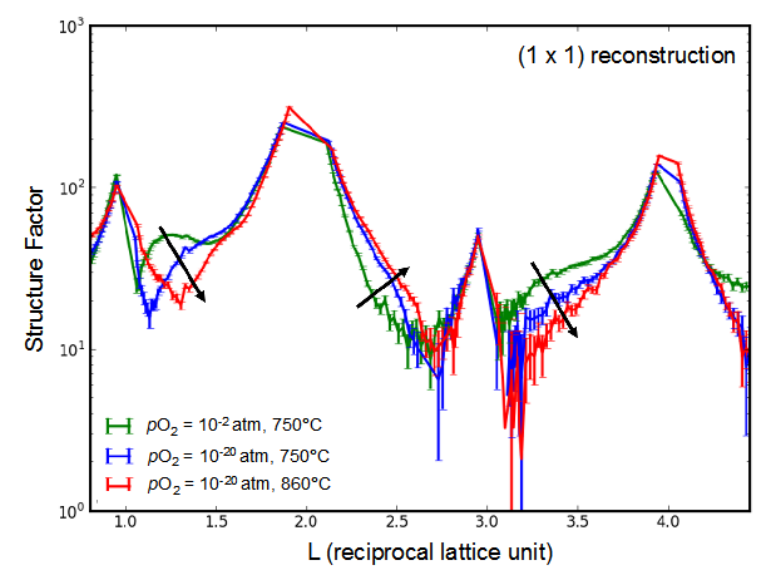

Figure 3: In-situ SXRD patterns of $\mathrm{SrTiO}_{3}(100)$.

temperatures and pressures.

As an example, Figure 3 shows out-of-plane SXRD patterns of $\mathrm{SrTiO}_{3}(100)$ perovskite oxide. Environmental conditions ranged from oxidizing $\left(\mathrm{O}_{2}\right)$ to reducing $\left(\mathrm{H}_{2} / \mathrm{H}_{2} \mathrm{O}\right)$ atmospheres and temperatures from 750 to $860^{\circ} \mathrm{C}$. A 1 × 1 reconstruction was observed, which was stable for more than 100 hours. Upon changing temperature and/or oxygen partial pressure, $\mathrm{SrTiO}_{3}$ exhibits pronounced changes in the SXRD pattern, suggesting relaxation in the out-of-plane atomic positions. These changes are likely associated with the formation of surface oxygen vacancies and/or emergence of $\mathrm{Ti}^{3+}$ electronic states.

\section{Publications}

None

\section{Workshops}

Y. Shi, Z. A. Feng, Y. Wen, M. F. Toney, W. C. Chueh.," Electrochemical activity of atomically flat ceria thin films of different orientations". Materials Research Society Spring Meeting, San Francisco, USA. Apr. 24, 2014.

W. C. Chueh. "Nonstoichiometric Oxide Surfaces Far From Equilibrium”. Materials Science \& Technology Conference, Oct. 29, 2013, Montreal, Canada.

W. C. Chueh. "Shining Light on Electrochemically-Active Materials". Denmark Technical University, Department of Energy Conversion \& Storage, Dec. 16, 2013, Copenhagen, Denmark.

W. C. Chueh." Ion Intercalation Reactions for Batteries E Fuel Cells". University of Wisconsin, Madison, Department of Materials Science \& Engineering, Apr. 17, 2014.

W. C. Chueh. "Dynamic Interaction of Surface Point Defects with $\mathrm{H}_{2} \mathrm{O}$ and $\mathrm{CO}_{2}$ in Cerium Oxide". European Materials Research Society Meeting, May 26, 2014, Lille, France.

W. C. Chueh. "Surface Redox Behavior of Metal Oxide Thin Films". Oxide Thin Films for Advanced Energy and Information Applications Conference, July 13, 2013, Chicago, USA.

W. C. Chueh. "Electrochemical Reactions on Oxygen-Deficient Oxide Surfaces". The 65th Meeting of the Solid State Ionics Society of Japan, Aug. 1, 2014, Sendai, Japan.

W. C. Chueh. "Ion Insertion Reactions in Fuel Cell Materials". HKUST-Caltech Workshop on Fuel Cells \& Electrolyzers, Aug. 19, 2014, Hong Kong, China. 


\section{Questionnaire}

\section{Question}

Will follow-on funding (post-LDRD project) be applied for?

Source of support for follow-on funding?

Has follow-on funding been obtained?

Amount of follow-on funding (\$K)?

Number of Post Docs supported by LDRD project?

Number of students supported by LDRD project?

Number of scientific staff/technical staff hired with LDRD funding?

Number of copyrights filed (beyond publications)?

Number of invention disclosures filed?

Number of patent applications filed?
Answer

Yes

DOE-BES Early

Career

No

$\$ 750$

\section{1}

0

0

0

0 


\subsection{Chemistry in motion: new approaches to probe enzymatic reaction mechanisms in crystallo}

Principal Investigator: Aina E. Cohen

\section{Project Description}

We will confront several experimental problems that are impeding progress in structural enzymology through the development of a toolbox of new experimental methods broadly applicable to the study of enzymatic reaction mechanisms in crystallo. A main scientific goal is to apply these technical innovations to trap several key high-valent intermediates in the mechanism of methane monooxygenase (sMMO), which have been predicted from solution studies, but have yet eluded structural characterization. One of the main inhibiting factors in determining catalytically accurate structures of intermediates with higher oxidation states has been the sensitivity of the bi-nuclear cluster to X-ray reduction. This ambitious endeavor to structurally characterize $\mathrm{Fe}(\mathrm{II})$-oxy, Fe(III)-peroxo and Fe(IV)-oxo intermediates of sMMO is a collaboration with Lipscomb (UMN). The information obtained would aid the development of biomimetic catalysts and may ultimately impact the use of methane as an alternative energy source. Other metalloenzymes that will be used as part of these collaborative studies include 1) extradiol aromatic ring-cleaving dioxygenase 2,3-HPCD (Lipscomb, UMN) and 2) dye-decolorizing peroxidase DypB (Eltis, UBC). The use of crystals is particularly beneficial to the analysis of catalytic reaction mechanisms due to physical characteristics imposed by the crystalline lattice that allows kinetic/mechanistic trapping and analysis of transient reaction intermediates that are short-lived in solution. Optical and vibrational spectroscopy will enable rapid and non-invasive identification, characterization and monitoring of these transient chemical species, providing information to optimize freeze-trapping experiments and to monitor degradation of these species while inside flow cells and injectors for X-ray studies. We will conduct this work on the bench and at the beamline to optimize the lifetimes of intermediates under varied conditions and use this information to adapt experimental designs for optimal performance. A particularly innovative aspect of these approaches will be the use of pressure techniques and flow cells in injectors to initiate and monitor reactions with gaseous substrates. It is imperative that our methodologies produce catalytically-relevant rather than radiation-affected structures. To this end, in situ spectroscopic monitoring at SSRL will determine the radiation 'lifetime' of crystals. Data from acutely radiation sensitive species will be collected using short X-ray pulses at LCLS (diffraction before destruction). When macrocrystals are available, these experiments will be conducted using the goniometer-based setup developed for LCLS XPP. For diffusion-limited reactions or when larger crystals are not available, serial femtosecond nano-crystallography experiments will be conducted at LCLS CXI.

\section{Accomplishments (year 1)}

The project began by focusing on sample crystallization and characterization as well as setting up new equipment for spectroscopic monitoring of reaction intermediates formed in crystallo inside flow cells and exiting the GDVN injectors. A number of LCLS beamtime proposals were 
submitted in relation to this project and a few hours of beam time at LCLS-XPP were awarded in mid-May 2014 for DypB and HPCD data collection, and one hour of nano-crystal screening time using the GDVN injector at LCLS CXI in July 2014. To ensure these crystals were in the proper intermediate state and of suitable diffraction quality, a sampling underwent spectroscopic characterization and diffraction quality screening at SSRL BL9-3, BL11-1 and BL12-2. Work is underway on processing and analysis of the diffraction data obtained. A summary of the progress made for each enzyme system is described in the sections below. Furthermore, a supply of PCD enzyme that easily forms showers of micro-crystals was also obtained, which will be used for initial testing of reaction initiation and monitoring within the injector setup to prepare for nanocrystallography experiments in the coming year.

sMMO: Crystallization of the MMOH-MMOB complex, with the aim of producing diffractionquality macro-crystals, has been the subject of significant effort for decades. Multi-variable screens were initiated to search for and to optimize production of nano- or micro-crystals of $\mathrm{MMOH}$ MMOB complex for our experiments. Multiple hits were identified that produce micro to nanometer protein crystals $(<5 \mu \mathrm{m})$ of potentially the MMOH-MMOB complex. One condition was screened at LCLS XPP in December 2013 and produced diffraction to $4 \AA$. Alternative crystallization conditions have been identified and are being optimized to improve the diffraction resolution.

DypB: Crystals of DypB were obtained in two crystal forms, one that diffracts to higher $(\sim 1.4 \AA)$ resolution. Parameters were optimized for the in crystallo reaction of DypB peroxidase to trap the high-valent $\mathrm{Fe}(\mathrm{IV})=\mathrm{O}$ intermediate. Successful trapping of the $\mathrm{Fe}(\mathrm{IV})=\mathrm{O}$ intermediate in DypB crystals was confirmed by X-ray Absorption Spectroscopy experiments at SSRL BL9-3, and by single crystal optical spectroscopy at SSRL BL11-1. Intermediate stability with X-ray dose was also evaluated. Structural analysis is in progress using a number of full XFEL diffraction datasets to better than $1.5 \AA$ resolution that were collected at LCLS XPP with crystals grown and reacted at different $\mathrm{pH}$ values. Preliminary results show evidence of non-photoreduced high-valent intermediate based on the refined Fe-O distances of 1.7 to $1.8 \AA$. Optimized data processing and data analysis are underway.

HPCD: Long crystals of 2,3-HPCDs have been obtained for X-ray diffraction studies using the goniometer setup at LCLS-XPP. XFEL diffraction datasets were collected from two HPCD variant/substrate complexes at LCLS-XPP producing one complete dataset to $1.5 \AA$ resolution and a $\sim 60 \%$ complete dataset. Data processing and analysis is underway. High-quality microcrystalline samples of HPCD were also produced and screened for diffraction quality using the GDVN injector at LCLS-CXI in July 2014, which resulted in over 90\% complete dataset to $2.0 \AA$ from $~ 5000$ usable diffraction images obtained in only $10 \mathrm{~min}$ of beam time. A manuscript describing microcrystal characterization using TEM and this "10-min structure" is in preparation. The positive results of these initial screening experiments clearly demonstrate the feasibility of the injector-based 'on-the-fly' approach to generate and structurally characterize transient reaction 
intermediates of HPCD in crystallo at LCLS-CXI. A follow-up beam time proposal has been submitted.

\section{Publications}

Stevenson, H. P., DePonte, D. P., Makhov, A. M., Conway, J. F., Zeldin, O. B., Boutet, S., Calero, G., and Cohen, A. E. "Transmission electron microscopy as a tool for nanocrystal characterization preand post-injector". Phil. Trans. R. Soc., 369, 1647 (2014)

Cohen, A.E., Soltis, S.M., González, A., Aguila, L., Alonso-Mori, R., Barnes, C.O., Baxter, E.L., Brehmer, W., Brewster, A.S., Brunger, A.T., Calero, G., Chang, J.F., Chollet, M., Ehrensberger, P., Eriksson, T.I., Feng, Y., Hattne, J., Hedman, B., Hollenbeck, M., Holton, J.M., Keable, S, Kobilka, S.B., Kovaleva, E.G., Kruse, A.C., Lemke, H., Lin, G., Lyubimov, A.Y., Manglik, A., Mathews, I.I., McPhillips, S.E., Nelson, S., Peters, J.W., Sauter, N.K., Smith, C.A., Song, J., Stevenson, H.P., Tsai, Y., Uervirojnangkoorn, M., Vinetsky, V., Wakatsuki, S., Weis, W.I., Zadvornyy, O.A., Zeldin, O.B., Zhu, D., Hodgson K.O. "Goniometer-based femtosecond crystallography” Proc. Natl. Acad. Sci. USA, accepted (2014)

Kovaleva, E.G., Stevenson, H. P., DePonte, A. M., Conway, Cohen, A. E., and Calero, G. "Crystal concentration and quality assessment for injector-based XFEL diffraction measurements" in preparation.

\section{Questionnaire}

\section{Question}

Will follow-on funding (post-LDRD project) be applied for?

Source of support for follow-on funding?

Has follow-on funding been obtained?

Amount of follow-on funding (\$K)?

Number of Post Docs supported by LDRD project?

Number of students supported by LDRD project?

Number of scientific staff/technical staff hired with LDRD funding?

Number of copyrights filed (beyond publications)?

Number of invention disclosures filed?

Number of patent applications filed?

\section{Answer}

Yes

$\mathrm{NIH}$

No

0

1

0

0

0 


\subsection{Center for Laboratory Astrophysics}

Principal Investigator: Siegfried H. Glenzer

\section{Project Description}

The goal of this LDRD is to develop laboratory astrophysics experiments delivering world-class $\mathrm{x}$ ray probing capabilities to some of the most important and difficult areas of astrophysical plasmas, i.e., cosmic particle acceleration, antimatter relativistic plasmas and high-pressure planetary physics. We will design accurate and first principles based experimental techniques to measure transient states and ultrafast phenomena, thus allowing critical experimental tests of theoretical predictions and simulations.. This is accomplished through preparing and designing high-power laser experiments on novel targets using particle-in-cell (PIC) simulations. In particular, the interaction of the future 200 TW laser at LCLS/SLAC with cryogenic hydrogen plasma jets is of particular interest. This new laser capability will be amended through cryogenic target development within this LDRD. At the end of the funding period the experiments at SLAC, complemented by detailed simulations, will allow us to reach the required regime to investigate relativistic interactions for particle acceleration and the early stages of collision less shock formation.

\section{Accomplishments}

Within the first year of funding we have built a new laser laboratory at SLAC to enable the development of novel continuous cryogenic jets and to perform high-power laser-matter interaction experiments. An example of the cryogenic jet development is shown in Figure 1. Our new design has achieved a reliable, spatially and temporally stable jet. This is of critical importance because for lasermatter interaction experiments we are focusing $\sim 5 \mathrm{~m}$ laser beams onto the jet for high repetition rate measurements. The main development leading to stable, small-diameter jets is the type of nozzle being used. Previous jets employed glass capillaries. Here, we work with thin metal platelets containing a drilled aperture. A small jet diameter of $5 \mu \mathrm{m}$ has been demonstrated in integrated experiments at the Matter in Extreme Conditions hutch at LCLS.

1D and 2D Particle-in-Cell (PIC) simulations at varying density and thickness have been performed to predict the interactions of the laser with the jet. The simulations use the fully relativistic code PICLS, and model laser

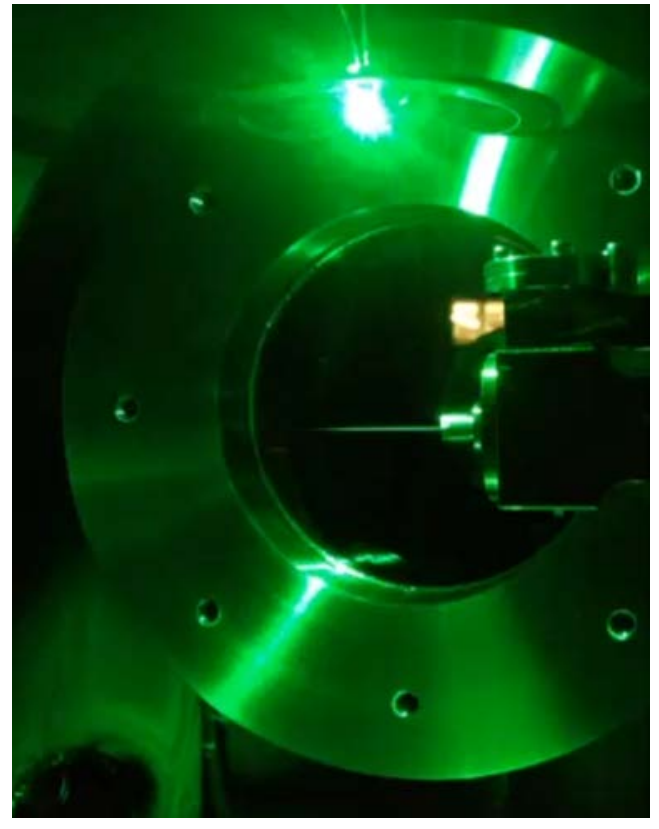

Figure 1: Image of cryogenic hydrogen jet expanding into vacuum at $100 \mathrm{~m} / \mathrm{s}$. The jet operating temperature of $15-20 \mathrm{~K}$, a vacuum pressure of $4 \times 10^{-4}$ resulted in 7 hour flow stability and $2 \mu \mathrm{m}$ pointing stability. 
intensities of $\sim 10^{20} \mathrm{~W} / \mathrm{cm}^{2}, 110$ fs duration with density of $6 \mathrm{n}_{\mathrm{c}}$ (where $\mathrm{n}_{\mathrm{c}}=10^{21} \mathrm{~cm}^{-3}$ ). The simulations show that the jet becomes relativistically under-dense for the laser and we observe that a strong (multi-terawatt) shock electric field is produced and protons are reflected to high velocities by this field. Further, the shock field and the laser field keep propagating through the hydrogen target and soon meet up with target normal sheath acceleration (TNSA) electric field produced at the target rear edge and vacuum interface and this superposition amplifies the TNSA fields resulting in increased proton energies. In addition, for transparent targets, the electrons at the rear edge continue to gain energy via strong interactions with the laser beam that propagates through the target. Figure 2 shows the results demonstrating high proton energies for micrometerscale hydrogen jets reaching levels of interest for applications in radiography, isochoric heating or tumor therapy.

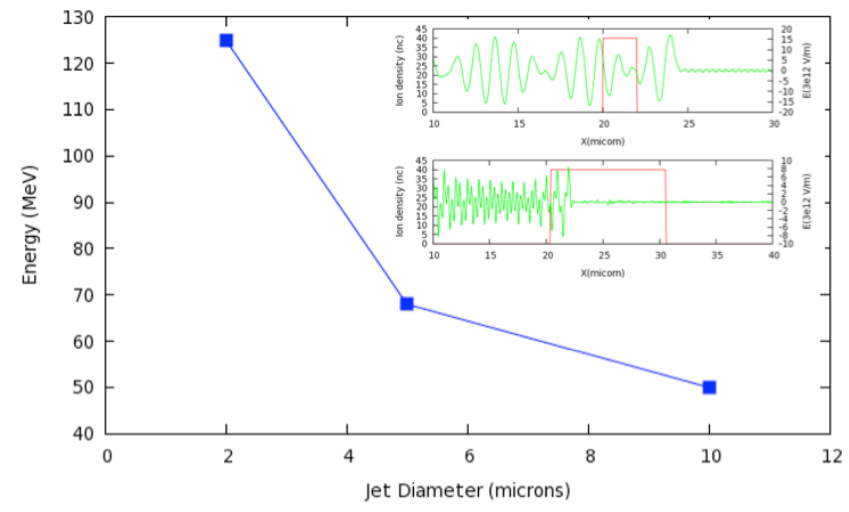

Figure 2: Computer simulations show the necessity for small diameter stable jets to achieve high energy proton beams. These simulations have been performed for a laser intensity of $2 \times 10^{20} \mathrm{~W} \mathrm{~cm}^{-2}$ in a $110 \mathrm{fs}$ long pulse. Our first test experiments have been performed at about $\sim 10 x$ lower intensity. The inset indicates that an increase in proton energy will be achieved in going to smaller jet diameters due to relativistic transparency.

To test laser-jet interaction physics we have commissioned a new laser laboratory in Sector-10 at SLAC. The figure shows the successful team with $25 \mathrm{~mJ}$ laser in the front, $40 \mathrm{fs}$ compressor, and a target chamber configured for pump-probe experiments.

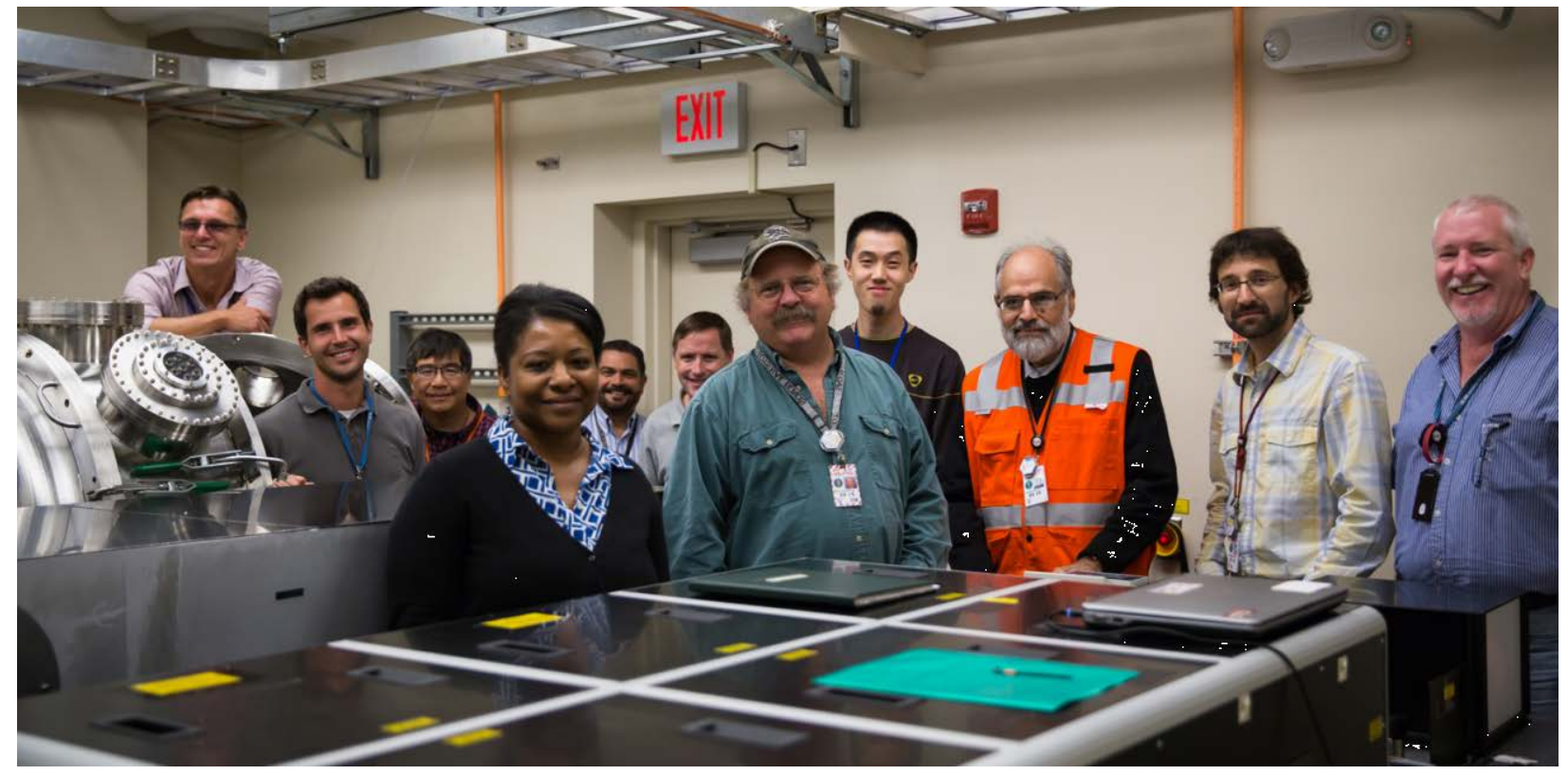

Figure 3: Completion of Sector-10 laser laboratory, October 232014. 


\section{Publications}

E. J. Gamboa, L. B. Fletcher, H. J. Lee, U. Zastrau, M. Gauthier, D. Gericke, J. Vorberger, E. Granados, P. Heimann, J. B. Hastings, and S. H. Glenzer, "Measurements of band gap structure in diamond compressed to $350 \mathrm{GPa}$ ", submitted to Phys. Rev. Lett. (2014).

H.-J. Lee, P. Sperling, E. J. Gamboa, H. K. Chung, Y. Omarbakiyeva, H. Reinholz, G. Roepke, U. Zastrau, L. B. Fletcher, and S. H. Glenzer, "Free-electron $x$-ray laser measurements of collisional-damped plasmons in isochorically heated warm dense matter", in preparation for Phys. Rev. Lett. (2014).

\section{Workshops}

R. Mishra, "Proton acceleration in the interaction of high power laser and cryogenic hydrogen targets", HPL-II workshop, Sponsored by DOE, Fusion Energy Sciences, Stanford CA, October 7-8, 2014.

P. Sperling, "Free-electron $x$-ray laser measurements of collisional-damped plasmons in isochorically heated warm dense matter", HPL-II workshop, Sponsored by DOE, Fusion Energy Sciences, Stanford CA, October 7-8, 2014.

R. Mishra, "Proton acceleration in the interaction of high power laser and cryogenic hydrogen targets", American Physical Society, Division of Plasma Physics meeting, New Orleans, LI October 27-31, 2014.

P. Sperling, "Free-electron $x$-ray laser measurements of collisional-damped plasmons in isochorically heated warm dense matter", American Physical Society, Division of Plasma Physics meeting, New Orleans, LI October 27-31, 2014.

\section{Questionnaire}

\section{Question}

Will follow-on funding (post-LDRD project) be applied for?

Source of support for follow-on funding?

Has follow-on funding been obtained?

Amount of follow-on funding $(\$ \mathrm{~K})$ ?

Number of Post Docs supported by LDRD project?

Number of students supported by LDRD project?

Number of scientific staff/technical staff hired with LDRD funding?

Number of copyrights filed (beyond publications)?

Number of invention disclosures filed?

Number of patent applications filed?

\section{Answer}

Yes

DOE (ARPA-E, etc.)

No

N/A

3

1

0

1

1

1 


\subsection{New Initiative for Synchrotron Radiation-based Catalysis and Energy Research}

Principal Investigator: Britt Hedman

\section{Project Description}

This LDRD project is focused in two areas, both related to synchrotron radiation-based chemical catalysis and energy research, using x-ray spectroscopy. One area is to develop high energyresolution x-ray emission techniques, coupled to theoretical calculations, to characterize the interactions between molecules and surfaces of catalysts under the operando conditions; the second area is to enhance characterization capabilities of catalytic materials through development of highthroughput techniques, applied to x-ray absorption (XAS) and emission (XES) measurements.

\section{Accomplishments}

High Energy-resolution X-ray Emission Techniques: The key element of the high energyresolution x-ray emission part of the LDRD has been the combination of the unique XES instrumentation on SSRL BL6-2 with construction and testing of sample cells to allow in-situ measurements under catalysis operando conditions. XES represents a highly sensitive tool to determine the electronic structure of metals, and how this structure changes during a reaction, providing structural information that can be applied to understanding the reaction mechanism. During the LDRD project, several cells designs were researched, and one designed and engineered that has the ability to withstand the required high temperatures and high pressure, and whose geometry enables both XES and XAS measurements. Several experiments that illustrate the use of x-ray emission spectroscopy were carried out in FY14, showing the feasibility of this spectroscopic approach and the information it provides. As a result, this technique is seeing a wider use by visiting scientists, with several projects at various stages of results.

High-throughput Technique Developments: The high-throughput spectroscopy developments, aimed at providing enhanced approaches for rapid characterization in catalysis and energy projects, using x-ray absorption spectroscopy, has focused on synergistically coupled hardware and software developments, with flexible implementations on the beam lines at SSRL. Building on developments throughout this LDRD project, (multiple-sample holding devices, alignment automation, software development), XAS data collection has become much faster, enabling higher throughput and faster rate of discovery for energy research on XAS beam lines.

Software developments have focused on flexible scripts that allow for direct automation of beam line optimization and data collection. This includes moving from one sample to the other in an automated fashion. Automated XAS data reduction and analysis, incorporating algorithms for determining the data quality while the sample is still being measured, provides direct feedback to the measurements. Software examines dynamically the quality of the data being measured, calculates statistics that is based on data noise levels, and enables the experimenter to automatically use the system intelligence to provide decisions as for when to advance to the next sample in the queue, thereby significantly improving data quality and experimental efficiency. 
This has been aided through the development of graphical user interfaces for easy display of results. The fundamental experimental and analysis tools have thus been implemented for highthroughput XAS characterization of materials. Development of a quick-scanning monochromator by SSRL progressed such that this instrument will be commissioned at the end of 2014 . This fastscanning monochromator, to be implemented on a refurbished beam line and serve SSRL and NSLS chemistry and catalysis users (during the transition period between NSLS and NSLS-II) will further enhance the high-throughput capabilities.

\section{Publications}

None

\section{Workshops}

None

\section{Questionnaire}

\section{Question}

Answer

Will follow-on funding (post-LDRD project) be applied for? Yes

Source of support for follow-on funding? DOE BES, Partners

Has follow-on funding been obtained? Yes

Amount of follow-on funding (\$K)? $\$ 450$

Number of Post Docs supported by LDRD project? 0

Number of students supported by LDRD project? 0

Number of scientific staff/technical staff hired with LDRD funding? 1

Number of copyrights filed (beyond publications)? 0

Number of invention disclosures filed? 0

Number of patent applications filed? 0 


\title{
7.0 Interfacial photoelectrochemistry using oxide heterostructures
}

\author{
Principal Investigator: Yasuyuki Hikita
}

\section{Project Description}

This project pursues the initiation of a new direction to establish a research platform for developing functional electrodes in photoelectrochemical applications using atomic scale controlled epitaxial oxide heterostructures. Exploiting the well-defined nature of our structures, the focus will primarily be to identify the critical factors in water photoelectrolysis and demonstrate new strategies to improve its efficiency based on electrostatic boundary conditions.

\section{Accomplishments}

We aimed at controlling the photoelectrode band alignments appropriately with respect to the hydrogen/oxygen evolution potential to provide larger thermodynamic driving force for electrons (holes) to produce hydrogen (oxygen). This involves forming surface dipole layers using ultrathin oxide thin films on top of the oxide photoelectrodes which will generate a sharp potential drop over atomic length scale. This year, the feasibility of this technique was examined in a model system consisting of ultrathin epitaxial oxide surface dipole layer on top of an oxide semiconductor in an all-solid device (Schottky junctions) using $\mathrm{Pt}$ as the top metal. From thorough characterization of the metal/semiconductor band alignment (Schottky barrier height, $\mathrm{SBH}$ ), we observed a linear decrease in the $\mathrm{SBH}$ over $0.8 \mathrm{eV}$ by depositing $\sim 1 \mathrm{~nm}$ of surface dipole layers. Based on this result, we will pursue the application of interface dipoles at oxide/electrolyte interfaces next year.

In addition to new approaches to improve the efficiency, we have also initiated a detailed study aiming at identifying factors limiting the solar photoelectrolysis efficiency. This year, we focused on the effect of particle size, which determines the semiconductor carrier mobilites. The use of epitaxial films enables to study the intrinsic particle size effect on the device efficiency by varying the thickness of the film while maintaining the same surface and bulk properties. We used anatase $\mathrm{TiO}_{2}$ epitaxial film with thickness varied between $34-83 \mathrm{~nm}$ as a model system. With increase in thickness, the incident photons to current efficiency (IPCE) increased in a step-wise manner, while the flat-band potential remained constant. The thickness at which the IPCE shows an abrupt increase corresponds with the thickness at which the lattice begins to relax, suggesting the importance of lattice strain on IPCE. We plan to do further experiments including optical characterization, doping dependence of IPCE, exploration for new active oxygen evolution catalysts.

\section{Publications}

None 


\section{Workshops}

Y. Hikita, "Manipulating band alignments at oxide heterojunctions using interface dipoles", MRS Spring Meeting 2014, San Francisco, CA, USA, April 21 - 25, 2014.

\section{Questionnaire}

\section{Question}

Will follow-on funding (post-LDRD project) be applied for?

Source of support for follow-on funding?

Has follow-on funding been obtained?

Amount of follow-on funding (\$K)?

Number of Post Docs supported by LDRD project?

Number of students supported by LDRD project?

Number of scientific staff/technical staff hired with LDRD funding?

Number of copyrights filed (beyond publications)?

Number of invention disclosures filed?

Number of patent applications filed?

\section{Answer}

Yes

Not determined yet

No

N/A

1

0

0

0

0

0 


\subsection{Towards in-Situ Growth and Spectroscopy of Complex Oxide Thin Films Heterostructures}

Principal Investigator: Harold Y. Hwang

\section{Project Description}

This project pursues the initiation of a new strategic direction within SLAC, to bridge advanced complex oxide thin film and heterostructure growth techniques with in situ characterization capabilities. A mobile pulsed laser deposition (PLD) system will be implemented to be compatible with surface chemistry, soft x-ray, and ARPES beam-lines at SSRL.

\section{Accomplishments}

A major goal of this LDRD was completed in the installation and operation of a new PLD growth system (Fig. 1), compatible with several SSRL beamlines. Installation was completed in May 2014, and over 100 films have been grown subsequently.

In parallel with this equipment development, we have been probing the microscopic origins of our unexpected finding of interface electronic structure control by surface adsorbates (Y. W. Xie, Y. Hikita, C. Bell, H. Y. Hwang, "Control of Electronic Conduction at an Oxide Heterointerface Using Surface Polar Adsorbates," Nature Communications 2, 494 (2011)). Using ambient pressure synchrotron X-ray photoemission spectroscopy, we studied the adsorption of water at the surface of the $\quad \mathrm{LaAlO}_{3} \quad / \mathrm{SrTiO}_{3}(100)$ heterostructure, a polar adsorbate which can

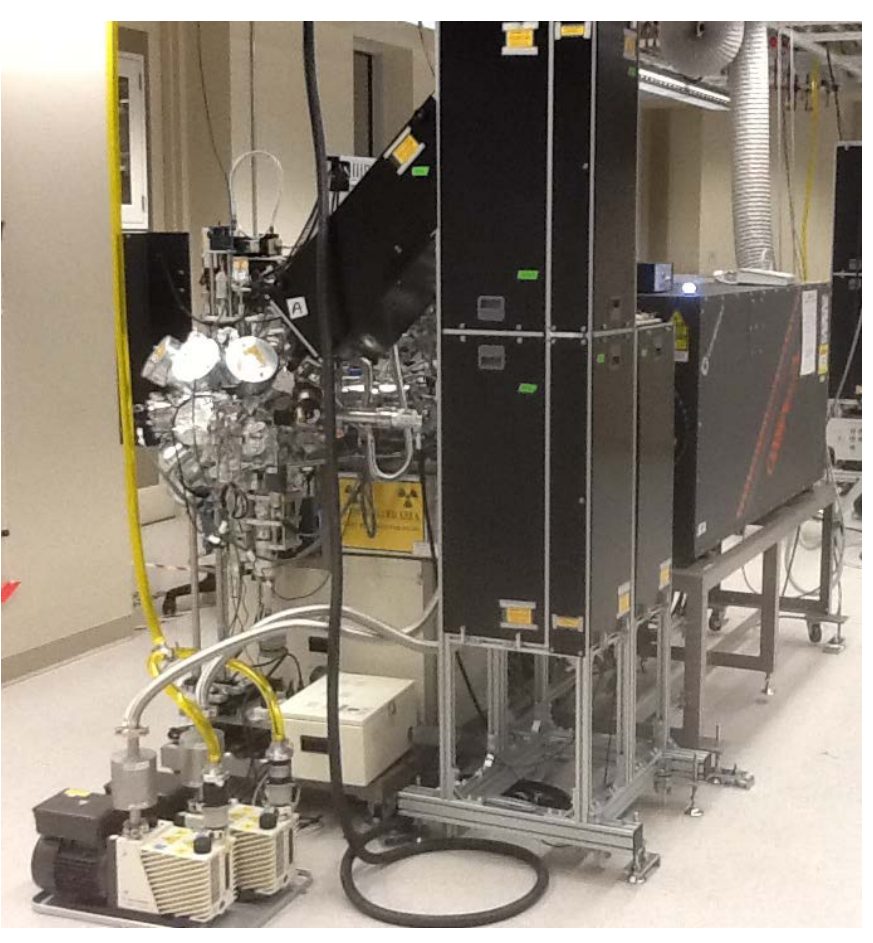

Fig. 1: Completed pulsed laser deposition system and integrated laser. induce a complete metal-insulator transition at the interface. $\mathrm{D}_{2} \mathrm{O}$ was used as the water source to minimize $\mathrm{X}$-ray induced dissociation of water. We found that, upon increasing the water vapor pressure $P$ from ultra-high vacuum to 1 Torr, the coverage of hydroxyl increases smoothly until saturation of 1 monolayer at $P \sim 10^{-2}$ Torr while the coverage of molecular water begins to increase abruptly for $P>10^{-4}$ Torr. In response to the adsorption of water, clear binding energy shifts of core levels of Al-2p, La- $4 d, \mathrm{O}-1$, and Sr-3d were observed. From these observations we conclude that water firstly adsorbs dissociatively at surface oxygen defects of the $\mathrm{AlO}_{2}$-terminated $\mathrm{LaAlO}_{3}(001)$ surface $\left(P<10^{-4}\right.$ Torr $)$, and then dissociates at surface $\mathrm{Al}$ atoms until saturation with a monolayer of hydroxyl, while molecular 
water is largely adsorbed on top of the hydroxyl groups. The dissociative adsorption at the surface $\mathrm{Al}$ atoms has the most important effect on the $\mathrm{LaAlO}_{3} / \mathrm{SrTiO}_{3}$ heterostructure, leading to a downward band bending up to $0.7 \mathrm{eV}$ across the $\mathrm{LaAlO}_{3}$ film.

\section{Publications}

Y. W. Xie, Y. Hikita, C. Bell, and H. Y. Hwang, "Adsorbate-Induced Insulator-Metal Transition in Oxide Heterostructures," in preparation.

Y. W. Xie, S. Kaya, H. Ogasawara, M. Minohara, Y. Hikita, C. Bell, A. Nilsson, and H. Y. Hwang, "Dissociative Adsorption of Water Decreases the Built-in Potential Across the $\mathrm{LaAlO}_{3} / \mathrm{SrTiO}_{3}$ Heterostructure," in preparation.

\section{Workshops}

H. Y. Hwang, "New Spectroscopic Measurements on Localized Charges at the Interface", American Physical Society March Meeting, Denver, CO, March 3-7, 2014.

H. Y. Hwang, "Reconstructions in Magnetic Oxide Heterostructures", 34th Los Alamos National Laboratory (LANL) Center for Nonlinear Studies (CNLS) Annual Conference on Mesoscale Science Frontiers, Santa Fe, NM, May 13-16, 2014.

\section{Questionnaire}

\section{Question}

Will follow-on funding (post-LDRD project) be applied for?

Source of support for follow-on funding?

Has follow-on funding been obtained?

Amount of follow-on funding (\$K)?

Number of Post Docs supported by LDRD project?

Number of students supported by LDRD project?

Number of scientific staff/technical staff hired with LDRD funding?

Number of copyrights filed (beyond publications)?

Number of invention disclosures filed?

Number of patent applications filed?

\section{Answer}

Yes

Not yet determined.

No

N/A

0

0

Partial funding for 1 assoc. staff scientist and PI.

0

0

0 


\subsection{KIPAC Inflation Initiative (CMB)}

Principal Investigator: Chao-Lin Kuo

\section{Project Description}

The goal of this program is to strengthen the current CMB program at SLAC through developing core technologies for CMB-S4, an ambitious ground-based survey aiming to extract full cosmological information contained in the polarization of the $\mathrm{CMB}$, and maintaining leadership in science programs.

In terms of core technologies for CMB-S4, we plan to expand our current effort in:

- Detector systems (multichroic polarimeters, SQUID multiplexers)

- Cold optics (infrared blocking filters, lenses, AR coating)

In terms of science programs, we maintain leadership/participation in:

- $\quad$ The BICEP/Keck program (Co-PI/PI institute)

- SPTpol and SPT-3G (cold optics, SQUIDs, cluster science)

- ACTpol and Advanced ACTpol (detector systems)

\section{Accomplishments}

The CMB effort at SLAC has been very small before the funding of this proposal. In 2013, the program grows substantially with the arrival of Kent Irwin's group and the funding of this proposal. In summer and fall of 2013, the SLAC CMB group worked closely with the CMB community to produce two Snowmass whitepapers that outlined the science and vision of CMBS4. These have received strong endorsements from the P5 (Particle Physics Project Prioritization Panel) in May. This significantly improves the prospect of programmatic return for this LDRD.

The main investments for the first fiscal year include:

- Cold optics (\$500k, BICEP3 and SPT-3G),

- Focal plane system (\$500k, BICEP3 detector modules),

- The funding profile was redistributed due to accelerated schedule for SPT-3G.

Project status and accomplishments:

- BICEP2 sees a significant B-mode at $150 \mathrm{GHz}$,

- BICEP3 (95GHz) fully tested, on track to deploy in 14/15 to South Pole to follow up BICEP2 results,

- Delivered two ACTpol polarimeter arrays (with NIST),

- Anti-reflection coating for SPT-3G developed

- Three alumina lenses purchased

- A Plan for metrology and anti-reflection coating in place. 


\section{Publications}

Ade, P. A. R. etal. "BICEP2 I: Detection of B-mode Polarization at Degree Scales using BICEP2, BICEP2 Collaboration", Phys. Rev. Lett. 112, 241101 (2014)

Ade, P. A. R. etal "BICEP2 II: Experiment and Three-Year Data Set, BICEP2 Collaboration", The Astrophysical Journal 792, 62 (2014)

Wu, W.L.K.; Errard, J.; Dvorkin, C.; Kuo, C.L.; Lee, A.; McDonald, P.; Slosar, A., and Zahn, O., “ $A$ Guide to Designing Future Ground-based CMB Experiments", The Astrophysical Journal, 788, 138 (2014).

Ahmed, Z.; Grayson, J.A.; Thompson, K.; Kuo, C. L.; Brooks, G; Pothoven, T., “Large-area Reflective Infrared Filters for Millimeter/sub-mm Telescopes", Journal of Low Temperature Physics, (2014).

\section{Workshops}

Ahmed, Z. etal."BICEP3: a 95 GHz refracting telescope for degree-scale CMB polarization", SPIE Astronomical Telescopes and Instrumentation 2014, Proceedings of SPIE Volume 9153, (2014) [refereed].

\section{Questionnaire}

\section{Question}

Answer

Will follow-on funding (post-LDRD project) be applied for? Yes

Source of support for follow-on funding? DOE, NSF, private

Has follow-on funding been obtained? No

Amount of follow-on funding (\$K)? N/A

Number of Post Docs supported by LDRD project? 2

Number of students supported by LDRD project? 3

Number of scientific staff/technical staff hired with LDRD funding? 0

Number of copyrights filed (beyond publications)? 0

Number of invention disclosures filed? 0

Number of patent applications filed? 0 


\subsection{Exploring the Scientific Capability of Momentum-Resolved Resonant Inelastic Soft X-ray Scattering for Material Science Research}

Principal Investigator: Wei-Sheng Lee

\section{Project Description}

An important approach to study the rich phenomena in strongly correlated quantum materials is to track elementary excitations associated with the underlying degrees of freedom. Recently, experiments using state-of-the-art RIXS instruments discovered a number of surprising behaviors of lattice, magnetic, and orbital excitations in these quantum materials, creating considerable excitements in the field. These excitements have motivated the synchrotron and x-ray free electron laser community worldwide to build next generation RIXS instruments with higher resolution and efficiency, promising to make significant impacts on future materials science research. Since SLAC is the home of two premier light sources (SSRL and LCLS) and commits to be a photon science center for materials science, this is a critical timing for SLAC to plan strategically in order to establish an on-site RIXS program. SIMES, the scientific drive for the forefront $x$-ray science at SLAC, is obligatory to lay the groundwork.

The goal of this proposal is to initiate a scientific RIXS program, which can further develop into a full-fledged program for materials science research, as well as serve as a driving force to realize RIXS instruments at SSRL and LCLS. We will attack important outstanding problems of quantum materials using state-of-the-art RIXS instruments worldwide. The publications and collaborations generated via this proposal will raise our scientific profile and develop the intellectual leadership in RIXS community. These activities are in conjunction with the commissioning of our q-RIXS endstation, expected in 2015 at ALS. The results of these commissioning experiments would be beneficial to develop LCLS proposals for time-resolved RIXS experiments at SXR, LCLS, expected in FY16.

\section{Accomplishments}

In FY14, the PI has started the project by applying for RIXS beamtime and performing preliminary experiments. These pieces of groundwork pave the road to expand the proposed research activities in full with the joint force of the postdoc scholar in FY15. The following list details the FY14 accomplishments:

Mapping out collective excitations in electron-doped cuprates: The PI has performed RIXS measurements to map out the doping evolution of magnetic and charge excitations in the stereotypical electron-doped cuprates, $\mathrm{Nd}_{2-x} \mathrm{Ce}_{x} \mathrm{CuO}_{4}$. We found that the behaviors of these collective excitations are notably different from those in their hole-doped counterparts. Some 
results are in a process of publication. The manuscript that systematically addresses their doping evolution is in preparation.

Recruiting a post-doctorate scholar: _We have interviewed six candidates and identified the most qualified one, Dr. Laura Chaix. She has finished her thesis defense and officially obtained her Ph. D. degree in September 2014, conferred by University of Joseph Fourier-Grenoble-I, France. We expect Dr. Chaix to arrive at SLAC in October/November 2014, pending on her VISA application process. We note that the reason why we cannot find a postdoc scholar to join us in FY14 is that the project starting time (Oct. 2013) did not match the usual job-hunting season (Jan.-Mar. 2013) for new Ph.D graduates. In FY14, we kept the spending of our project to a minimum, such that the fund can be carried over to FY16 to cover the two-year salary of Dr. Chaix. With the joint force of Dr. Chaix, we expect the project will proceed in full speed in FY15 and FY16.

Gaining access to state-of-the-art RIXS instruments: In FY14, the PI has started to apply for beamtime that is crucial to conduct this project. Until now, we have successfully obtained RIXS beamtime at Swiss light source (SLS, Switzerland) and Nation Synchrotron Radiation Research Center (NSRRC, Taiwan), where RIXS spectrometers with presently highest energy resolution at the $\mathrm{Cu}$ L-edge are available. The PI has also submitted beamtime proposals to ALS, NSRRC, SLS, APS, and ESRF to applying for beamtime in FY15. We expect more RIXS beamtime in FY15, aligning well with Dr. Chaix's joint force.

\section{Project Plan for FY15 and FY16}

Scientifically, we plan to continue explore the collective excitations in high Tc cuprates. In particular, we will focus on the following three problems: (a) how do collective excitations change across phase boundaries and putative quantum critical points? (b) How can we extract momentum-dependent electron-phonon coupling strength using the unique capability of RIXS? (c) How do translation-symmetry-broken states, such as spin and charge orders, couple to elementary excitations? In addition, we also plan to conduct experiments on novel quantum materials, including transition metal diachalcogenides (e.g. MoSe 2 and WSe2) and oxide heterostructures.

Technically, we will continue applying for beamtime of state-of-the-art RIXS instruments worldwide to carry out our scientific research. In addition to SLS and NSRRC, we have also formed collaborations with the RIXS group in ESRF where a new soft x-ray RIXS instrument with an energy resolution better than $50 \mathrm{meV}$ at $\mathrm{Cu}$ L-edge will be available in year 2015. In parallel, our FWP team expects to complete the q-RIXS endstation before the end of 2014. We plan to conduct commissioning experiments at ALS in 2015. The results of these commissioning experiments would be beneficial to develop LCLS proposals for time-resolved RIXS experiments at SXR, LCLS, expected in FY16.

\section{Publications}

In Preparation. 


\section{Workshops}

W. S. Lee, "Lattice, magnetic, and charge excitation in Cuprates", High resolution RIXS and Nano ARPES workshop, Sponsored by National synchrotron Radiation Center, Hsinchu, Taiwan, September 10-12, 2014.

W. S. Lee, "Lattice, magnetic, and charge excitations in Cuprates", APS March Meeting, Denver, CO, USA, Sponsored by American Physical Society, March 3-7, 2014.

\section{Questionnaire}

Question

Will follow-on funding (post-LDRD project) be applied for?

Source of support for follow-on funding?

Has follow-on funding been obtained?

Amount of follow-on funding (\$K)?

Number of Post Docs supported by LDRD project?

Number of students supported by LDRD project?

Number of scientific staff/technical staff hired with LDRD funding?

Number of copyrights filed (beyond publications)?

Number of invention disclosures filed?

Number of patent applications filed?

\section{Answer}

Yes

DOE/Early Career

Proposal

No

$\mathrm{N} / \mathrm{A}$

1

0

0

0

0

0

Notes on number of post-docs/students/staff: Any post-doc or student who was supported for more than $10 \%$ of his/her time should be included here. For staff, this is for staff that were hired specifically for and funded by the LDRD project (at least $50 \%$ of their time). 


\subsection{Low Dimensional Materials for Energy Applications}

Principal Investigator: Robert G. Moore

\section{Project Description}

The goal of this project is to explore low dimensional quantum oxide and chalcogenide materials and heterostructures with potential energy applications to understand the fundamental scientific processes connecting atomic and electronic structures in reduced dimensions. The past decade has seen revolutionary advances in the precise control of thin films and heterostructures on the atomic scale. Many new emergent states have been discovered but the underlying electronic structure governing these phenomena is unknown. Coupling thin film growth systems to state of the art angle resolved photoemission spectroscopy facilities will advance the understanding of emergent states and allow for rapid feedback in developing materials with tailored properties. This project is to develop a new strategic direction at SLAC for utilizing thin film growth and electron spectroscopy, both ex-situ and in-situ, to illuminate underlying material processes and foster creative material science solutions in material design.

\section{Accomplishments}

Capitalizing on recent DOE instrumentation investment in a thin film growth system, during FY14 we commissioned a Veeco GEN930 oxide MBE system and a DCA ozone distillation unit. These instruments will be coupled to a new photoemission beamline that will be commissioned by the end of the calendar year. Characterization of the growth systems has been completed and the development of $\mathrm{SrTiO}_{3}$, and $\mathrm{TiO}_{2}$ thin films with transition metal dopants and oxygen-18 is underway. Preliminary results from this system suggest an isotope effect in superconducting FeSe monolayers grown on $\mathrm{SrTiO}_{3}$ with oxygen-18. Such results are key to revealing the transition temperature enhancement is due to coupling between the chalcogenide film and a phonon mode in the oxide substrate. Growth of materials as $\mathrm{BaTiO}_{3}, \mathrm{La}_{2}-\mathrm{S} \mathrm{Sr}_{x} \mathrm{CuO}_{2}$ and $\mathrm{La}_{2}-\mathrm{Ba} \times \mathrm{CuO}_{2}$ to study superconductivity in the 2D limit and interface coupling between a ferroelectric and a superconductor is currently underway.

This project has also supported the development of a second growth chamber tailored to the growth of chalcogenide materials. This growth system will also be coupled to the beamline infrastructure. Oxide and chalcogenide materials cannot be grown in the same growth chamber as cross contamination of $\mathrm{SeO}_{2}$ molecules can contaminate vacuum systems. The second chamber will support the growth of materials related to topological insulators, thermoelectric applications and recently discovered $\mathrm{MX}_{2}$ direct bandgap semiconductors. Coupling the oxide and chalcogenide chambers via sample transfer system will allow for the development of oxide/chalcogenide heterostructures as $\mathrm{FeSe}_{\mathrm{SrTiO}}$ for the development of enhanced superconductivity in these systems. This second growth chamber has been designed and is currently in the procurement phase. This system will be completed and fully commissioned by second quarter of FY15. 
This project has also supported the development of an in situ four point transport probe to be incorporated into our ARPES system. It is difficult to measure transport properties of materials a single unit cell thick. Once removed from the vacuum system, atmospheric contamination can change the properties of materials of this thickness. Any attempts to cap the film with a protective layer can also alter the physical properties of such a thin film. Thus ex-situ transport measurements are typically unreliable. We have developed an in-situ four point transport probe that will allow accurate measurements of macroscopic transport properties of films down to thicknesses of a few angstroms. The probe has been constructed and is currently being commissioned. Preliminary results on bulk crystals show superior performance with excellent signal to noise ratio. The probe will be incorporated into our ARPES chamber by the end of the calendar year.

\section{Publications}

None.

\section{Workshops}

R. G. Moore, "Interfacial Interplay Between Superconducting FeSe Films and Underlying Substrate", American Physical Society Annual March Meeting, March 3-7, 2014, Denver, CO.

R. G. Moore, "Growth and in-situ Investigation of the Electronic Structure of Low Dimensional Transition-Metal Complexes", Stanford Synchrotron Radiation Lightsource Annual User's Meeting, October 7-10 2014, Menlo Park, CA

\section{Questionnaire}

\section{Question}

Will follow-on funding (post-LDRD project) be applied for?

Source of support for follow-on funding?

Has follow-on funding been obtained?

Amount of follow-on funding $(\$ \mathrm{~K})$ ?

Number of Post Docs supported by LDRD project?

Number of students supported by LDRD project?

Number of scientific staff/technical staff hired with LDRD funding?

Number of copyrights filed (beyond publications)?

Number of invention disclosures filed?

Number of patent applications filed?

\section{Answer}

Yes

DOE-BES

No

N/A

0

0

0

0

0 


\subsection{Screening of Alloy Catalysts for Electrochemical $\mathrm{CO}_{2}$ Reduction}

PIs: Jens K. Nørskov, Anders Nilsson, Thomas F. Jaramillo

\section{Project Description}

Photo-electrochemical reduction of $\mathrm{CO}_{2}$ to fuels (artificial photosynthesis) is one of the grand challenges to science and engineering. It has all the important ingredients: a very hard problem with the possibility of leading to game-changing technology, if successful. The main problem is the complete lack of suitable catalysts - few materials work at all and all are extremely inefficient (high overpotentials). At SUNCAT we have worked to analyze theoretically the best known catalyst, $\mathrm{Cu}$, to understand how it works. On this basis we have developed a model of the process, which allows us to understand trends in catalytic activity from one metal to the next. The model points to the fundamental reason why no single-component metallic catalysts have been found. The model also suggests possible strategies for changing the surface properties to achieve lower overpotentials. One possibility is to have two components in the active site at the surface, and recently we have started computationally to screen for new alloy catalysts. It is essential to couple this to synthesis, characterization, and test of new ideas. This should provide feedback to the theory and ultimately provide experimental proof of the existence of new catalysts.

\section{Accomplishments}

Design of a Vacuum Physical Vapor Deposition System: In order to provide feedback to theory with a tight development cycle, we have designed a PVD system with several requirements in mind. (1) The method should be able to co-deposit elements with in situ monitoring of the deposition rate. (2) The deposition method must be compatible and work immediately with a wide range of elements. (3) Precursor sources should require little monetary investment. (4) The entire deposition process must have a throughput that allows for daily electrochemical testing. Using these criteria, we chose a vacuum evaporation system rather than a sputtering system, because in situ deposition rate monitoring can be achieved immediately for a wide range of elements in a vacuum evaporation system by using a quartz crystal microbalance (QCM). While atomic absorption spectroscopy can be used to monitor the deposition rate during sputtering, a separate calibration curve is required for each element. Therefore, a vacuum evaporation system better fits our demand for a medium-throughput synthetic method.

Together with Technical Engineering Services during the spring of 2013, we designed a dual ebeam thermal evaporator system that can co-deposit up to three elemental sources simultaneously onto a 4" wafer. The deposition rate of all three sources can be monitored and adjusted through a feedback loop between each QCM and the deposition controller. The vacuum deposition chamber is also equipped with a quartz lamp to allow for in situ heating of the sample. The PVD system was successfully installed and calibrated during the summer of 2014. 

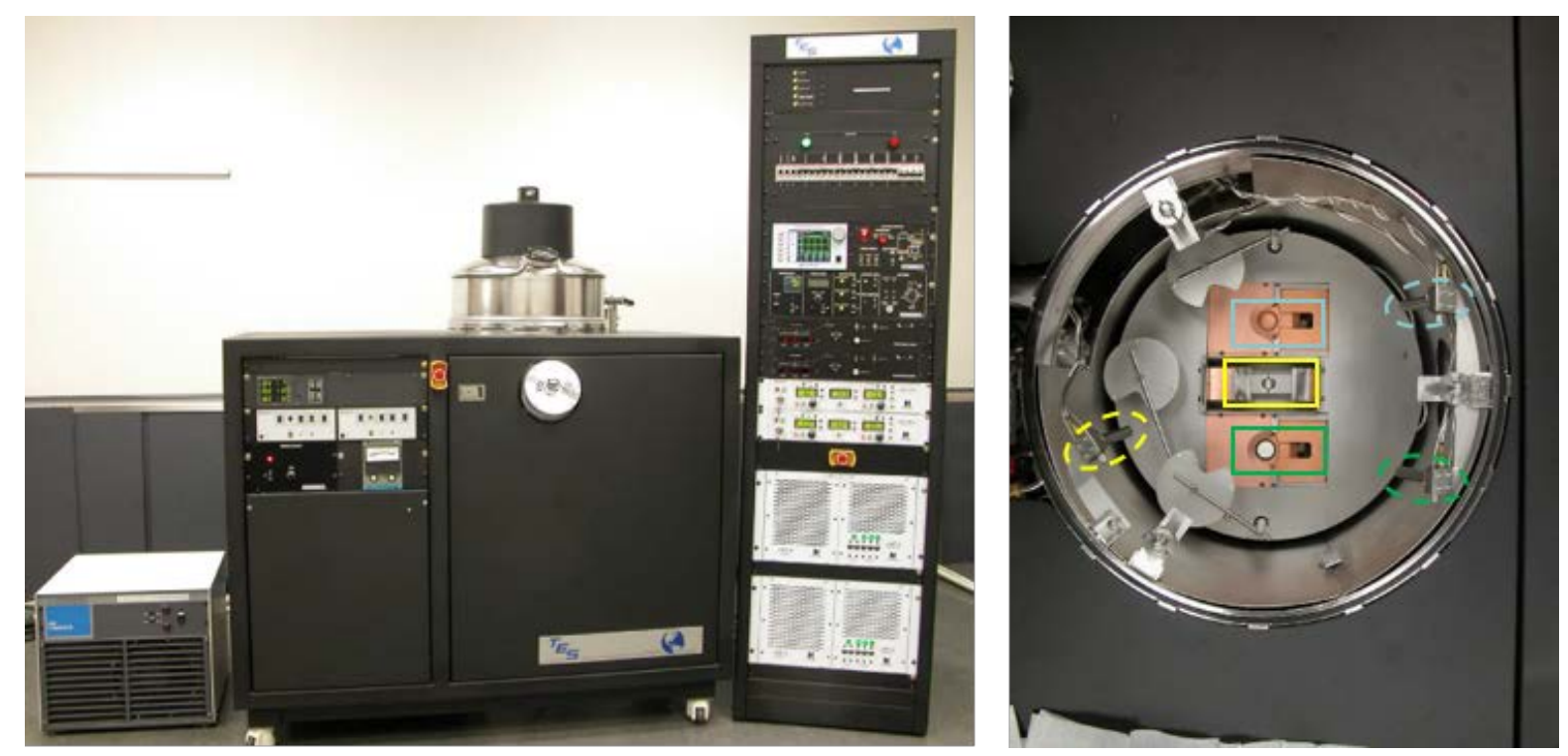

Figure 1: Fully assembled three source PVD system (left). Each source (solid rectangle) has its own corresponding QCM (dashed oval) for deposition rate control.

Au1-ydy Thin Film Synthesis and Characterization: In anticipation of the arrival of the new PVD system, we developed protocols for the synthesis of binary alloy thin films at other facilities with the capability of co-deposition. The nanofabrication facility at UCSB has a dual e-beam evaporator that can co-deposit up to two elements. Each e-beam has a QCM that can monitor the deposition rate from the source, allowing for experimental control over the composition of the thin film. Out of the possible alloys that were available to deposit on this instrument, our calculations show that $\mathrm{Au}_{1-\mathrm{y}} \mathrm{Pd}_{\mathrm{y}}$ could have one of the most favorable limiting potentials for $\mathrm{CO}_{2}$ reduction. Therefore, we have conducted a detailed study of this alloy system by depositing a broad range of compositions.

Figure 2: X-ray diffractograms demonstrate tuning of the bulk composition of $A u_{1-y} P d_{y}$ alloys. The Pd composition increases from left to right.

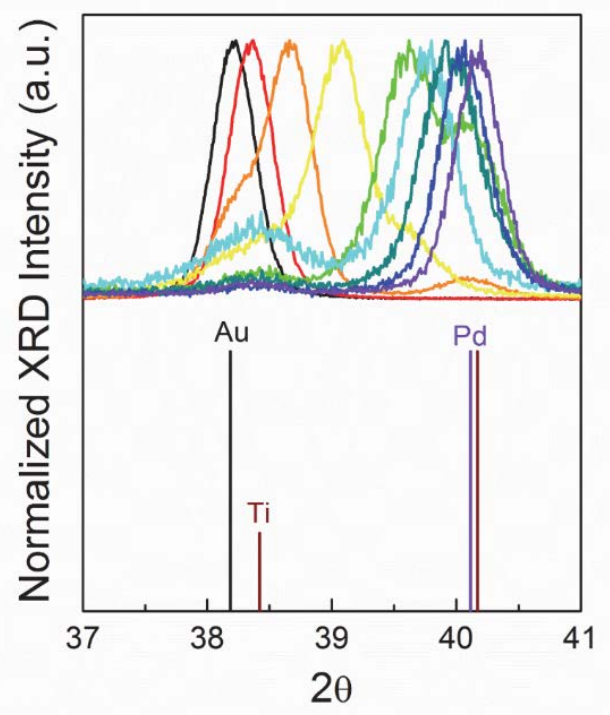



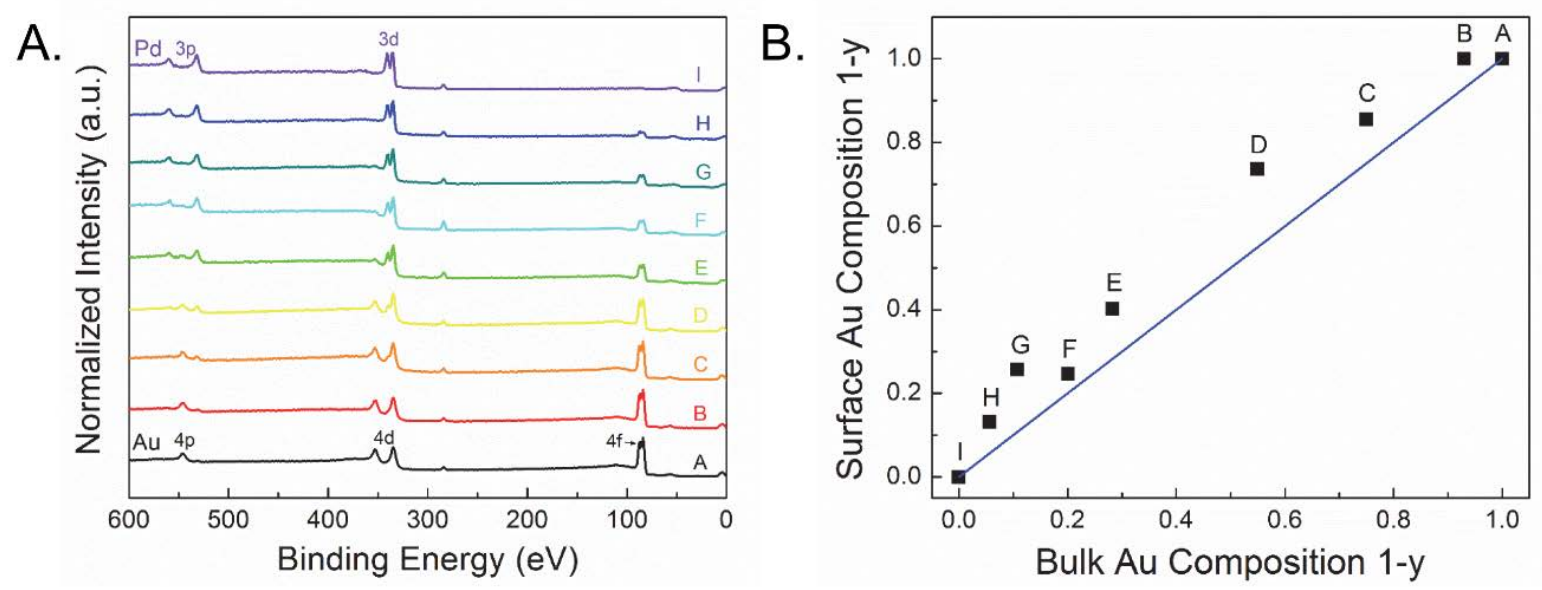

Figure 3: X-ray photoelectron spectra demonstrate tuning of the surface composition of $A u_{1-y} P d_{y}$ alloys. The $P d$ composition increases from bottom to top. (B) A comparison of bulk and surface Au compositions of $A u_{1-y} P d_{y}$ alloys shows that the surface is slightly Au-rich. The blue line represents a surface to bulk composition ratio of 1 .

Bulk and surface compositions of $\mathrm{Au}_{1-\mathrm{y}} \mathrm{Pd}$ y thin films were characterized using $\mathrm{x}$-ray diffraction (XRD) and x-ray photoelectron spectroscopy (XPS), respectively. Both Au and Pd adopt the facecentered cubic (FCC) crystal structure but have a large lattice mismatch of $4.6 \%$. XRD patterns in Figure 2 clearly show a shift of the $\mathrm{Au}_{1-\mathrm{y}} \mathrm{Pd}_{\mathrm{y}}$ FCC (111) peak towards larger diffraction angles from an increase in Pd composition. This shift in the lattice constant demonstrates that $\mathrm{Au}$ and Pd codeposit as an alloy rather than pure $\mathrm{Au}$ and Pd, indicating that our PVD approach will work for even lattice mismatched material systems. While XRD spectra demonstrate alloying within the bulk of $\mathrm{Au}_{1-\mathrm{y}} \mathrm{Pd}_{\mathrm{y}}$ thin films, experimental control of the surface composition is also critical because electrochemical $\mathrm{CO}_{2}$ reduction occurs on the surface of the alloy catalysts. XPS spectra show a similar trend in composition on the surface (Figure 3A) when compared to the bulk, indicating that the surface composition of $\mathrm{Au}_{1-\mathrm{y}} \mathrm{Pd}_{\mathrm{y}}$ thin films can also be tuned by PVD. A comparison of the Vegard's law bulk Pd composition and the XPS surface Pd composition shows that the $\mathrm{Au}_{1-\mathrm{y}} \mathrm{Pd}_{\mathrm{y}}$ thin films are slightly Au-rich on the surface (Figure 3B).
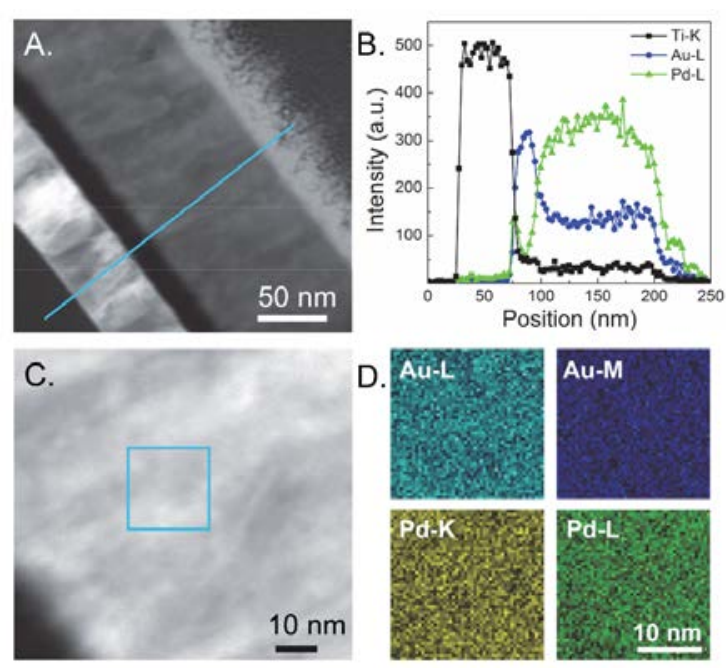

Figure 4: (A) A dark field scanning transmission electron microscope image shows the cross section of a representative AuPd thin film. (B) An energy dispersive $x$-ray spectroscopy line scan shows that the composition of the AuPd layer is relatively uniform along the cross section. $(C, D)$ Further analysis by mapping $A u$ and Pd signals demonstrates that the composition of the alloy is uniform on a nanometer scale.

Scanning transmission electron microscopy (STEM) was used to examine the composition of a representative AuPd thin film on the nanometer scale. A cross-section of AuPd was cut with a Ga focused ion beam and subsequently transferred and bonded to a TEM grid using a micromanipulator. A 
dark-field STEM image of the cross-section can be seen in Figure 4A. The blue line represents the region where an energy dispersive $x$-ray spectroscopy (EDS) line scan measured the composition. The corresponding $x$-ray fluorescence intensities for the Ti binding layer, $\mathrm{Au}$, and $\mathrm{Pd}$ (Figure 4B) show a sharp interface between the Ti binding layer and the AuPd thin film. The peaks in Au and $\mathrm{Pd}$ intensities, at the interface with $\mathrm{Ti}$, represent the region where the evaporation sources were individually calibrated to prevent cross talk with the QCMs. Further analysis using EDS mapping (Figures $4 \mathrm{C}, \mathrm{D}$ ) demonstrates that $\mathrm{Au}$ and $\mathrm{Pd}$ are distributed uniformly within the alloy. These results indicate that e-beam co-deposited thin films can be uniform in composition on the nanometer scale, and therefore provide an excellent platform for fundamental electrochemical studies.

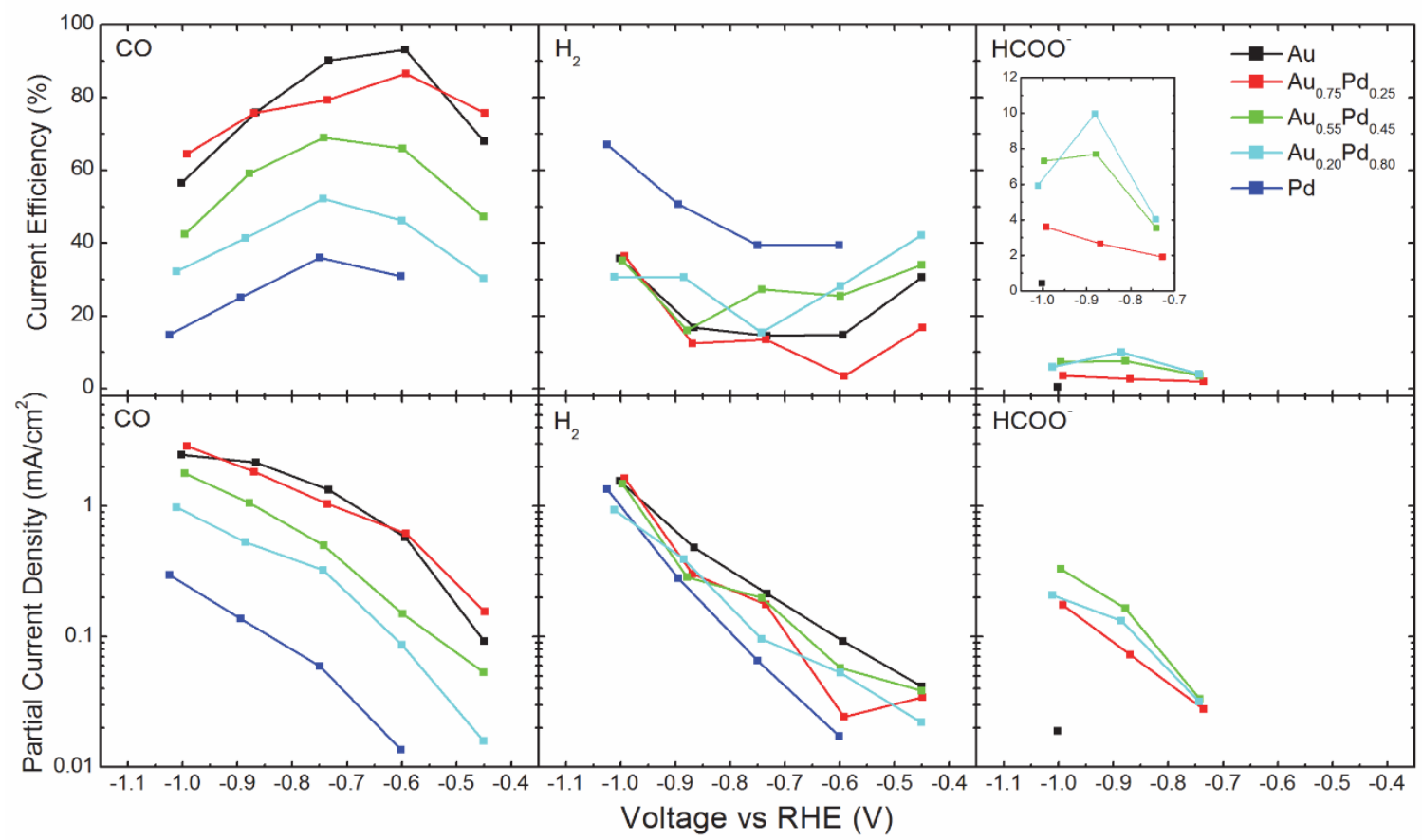

Figure 5: Current efficiencies (top) and partial current densities (bottom) for $\mathrm{CO}, \mathrm{H}_{2}$, and HCOO- are plotted as a function of voltage for a range of $A u_{1-y} P d_{y}$ compositions.

The $\mathrm{CO}_{2}$ reduction activity and selectivity of $\mathrm{Au}_{1-y} \mathrm{Pd}_{\mathrm{y}}$ thin films were examined using electrochemical measurements in tandem with gas phase (gas chromatography) and liquid phase (nuclear magnetic resonance) product detection methods. Working electrodes were held at a fixed potential for one hour while the total current was measured. Multiple $\mathrm{Au}_{1-\mathrm{y}} \mathrm{Pd}_{\mathrm{y}}$ compositions were measured to determine the dependence of activity on the composition of the electrode. The current efficiencies and partial current densities for $\mathrm{CO}_{2}$ reduction to $\mathrm{CO}, \mathrm{H}_{2}$, and $\mathrm{HCOO}^{-}$are compared in Figure 5. While the $\mathrm{Au} 0.75 \mathrm{Pd}_{0.25}$ alloy has a similar activity and selectivity as pure $\mathrm{Au}$ for $\mathrm{CO}$ production, an increase in $\mathrm{Pd}$ composition causes the activity and selectivity to decrease. Surprisingly, $\mathrm{Pd}$ produces lower partial current densities for $\mathrm{H}_{2}$ than the alloys although it has a higher current efficiency for $\mathrm{H}_{2}$. One reason for the lower partial current densities could be $\mathrm{CO}$ poisoning of active sites, since Pd binds CO stronger than Au does. All alloy compositions have a 
significantly higher current efficiencies and partial current densities for HCOO- than pure Au or $\mathrm{Pd}$. One possible reason for the enhancement in formate production could be an increase in metalhydride species from hydrogen intercalation into the Pd lattice. This increase in local hydrogen coverage could facilitate hydrogenation of an adsorbed $\mathrm{COO}^{-}$molecule on an adjacent $\mathrm{Au}$ site. These measurements clearly demonstrate that alloying can have synergistic effects to yield new electrocatalytic properties.

Binary Alloy Screening: Since the summer of 2014, we have successfully installed and fully integrated the three source PVD system within our alloy screening efforts. Using theory to help guide us, we've examined alloys ranging from weak $\mathrm{CO}$ binding (AuAg, AuIn), to intermediate $\mathrm{CO}$ binding (CuNi, CuPt, CuAu, CuAg, AuPd, AuTi, AuCr, PtIn), to strong CO binding (PtCo). In particular, we've observed some interesting synergistic effects from alloying $\mathrm{Pt}$ and $\mathrm{In}$, a combination of a transition and a p-block metal. Initial electrochemical tests on PtIng showed that it is more selective and active for $\mathrm{CO}$ than either pure Pt or In. Afterwards, several different compositions of PtIn were synthesized to determine its effect on activity and selectivity. Current efficiencies (Figure 6) show that all In-rich alloys (PtIn4, PtIn7, and PtIn19) are more selective for CO than either pure $\mathrm{Pt}$ or $\mathrm{In}$, indicating that alloying of $\mathrm{Pt}$ and In is engendering new electrocatalytic behavior. The PtIn7 alloy is the most selective composition for $\mathrm{CO}$, with a maximum current efficiency of $49 \%$ at $-0.68 \mathrm{~V}$ vs RHE. In addition, interesting trends in selectivity are observed with $\mathrm{H}_{2}, \mathrm{CO}$, and $\mathrm{HCOO}^{-}$sequentially becoming the major product as the voltage is increased. While the nature of $2 \mathrm{e}^{-}$product selectivity is still ambiguous, ongoing theoretical efforts will help to elucidate these trends.

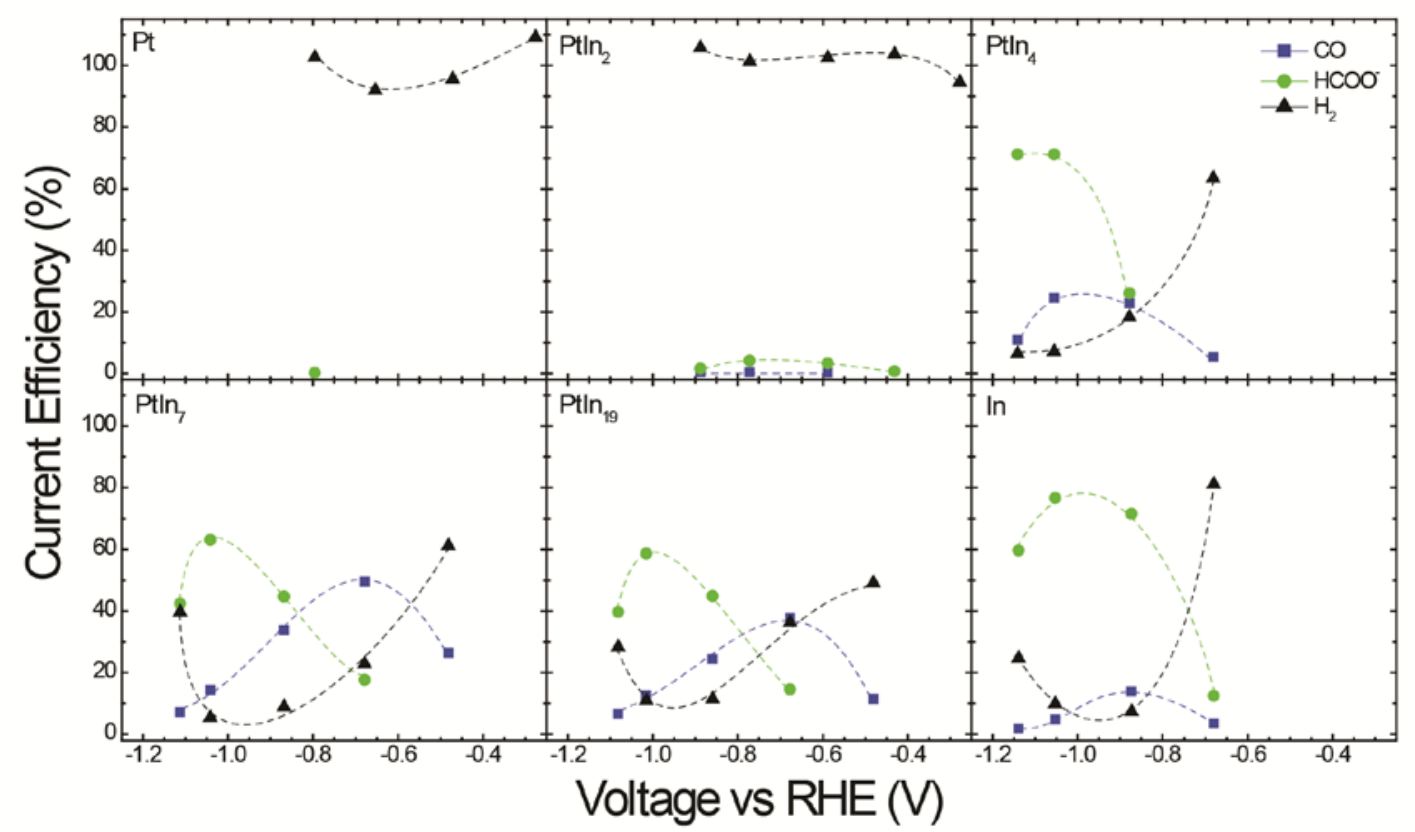

Figure 6: Current efficiencies for $\mathrm{CO}, \mathrm{HCOO}$, and $\mathrm{H}_{2}$ are plotted as a function of voltage, separated by PtIn alloy composition. Samples that are In-rich show greater selectivity to CO than either pure Pt or In at lower overpotentials. 
Conclusions: In conclusion, we have successfully designed, procured, and integrated a three source PVD system for alloy thin film synthesis. Characterization of the structure and bulk/surface compositions of AuPd thin films indicates that we have established a robust PVD method to synthesize a targeted library of alloys. Screening of electrocatalysts yielded two systems, AuPd and PtIn, which have shown synergistic electrocatalytic effects from alloying. Based on the tools, methods, and science developed through this project, we are embarking on new, expanded studies for the discovery and development of new alloy electrocatalysts.

\section{Publications}

Tang, M.H.; Hahn, C.; Klobuchar, A.J.; Ng, J.W.D.; Wellendorff, J.; Bligaard, T.; Jaramillo, T.F., "Nickel-silver alloy electrocatalysts for hydrogen evolution and oxidation in an alkaline electrolyte," Physical Chemistry Chemical Physics, 16, 19250-19257, 2014.

Hahn, C.; Abram, D.N.; Hansen, H.A.; Hatsukade, T.; Jackson, A.; Johnson, N.C.; Hellstern, T.R.; Kuhl, K.P.; Cave, E.R.; Feaster, J.T.; Jaramillo, T.F., "Synthesis of Thin Film AuPd Alloys and their Investigation for Electrocatalytic $\mathrm{CO}_{2}$ Reduction," Submitted, 2014.

Abram, D.N.*; Hahn, C. ; Hansen, H.A.; Shi, C.; Hatsukade, T.; Kuhl, K.P.; Cave, E.R.; Feaster, J.T.; Nilsson, A.; Nørskov, J.K.; Jaramillo, T.F., "Synergistic Electrocatalytic $\mathrm{CO}_{2}$ Reduction Properties of PtIn Alloy Thin Films," In Preparation, 2014.

Hahn, C.*; Abram, D.N.*; Hansen, H.A.; Shi, C.; Hatsukade, T.; Kuhl, K.P.; Cave, E.R.; Feaster, J.T.; Nilsson, A.; Nørskov, J.K.; Jaramillo, T.F., "Screening Binary Alloy Catalysts for Electrochemical $\mathrm{CO}_{2}$ Reduction," In Preparation, 2014.

\section{Workshops}

Jaramillo, T.F. "Design Principles for Catalyzing $\mathrm{CO}_{2}$ Electro-Reduction to Fuels and Chemicals on Transition Metal Surfaces," Annual Global Climate and Energy Project Research Symposium, Stanford, CA, October 2014.

Hahn, C.; Abram, D.N.; Hansen, H.A.; Hatsukade, T.; Kuhl, K.P.; Feaster, J.T.; Cave, E.R.; Nilsson, A.; Nørskov, J.K.; Jaramillo, T.F., "Physical Vapor Deposition of Binary Alloys for Electrochemical $\mathrm{CO}_{2}$ Reduction," Materials Research Society Spring Meeting, San Francisco, CA, April 2014.

Tang, M.H.; Hahn, C.; Ng, D.; Jaramillo, T.F., "Metastable Nickel-Silver Alloy Electrocatalysts for Alkaline Hydrogen Evolution and Oxidation," Materials Research Society Spring Meeting, San Francisco, CA, April 2014.

Tang, M.H.; Hahn, C.; Ng, D.; Jaramillo, T.F., “Non-Noble Electrocatalysts for Alkaline Hydrogen Evolution and Oxidation," Electrochemical Society Meeting, San Francisco, CA, October 2013. 


\section{Questionnaire}

\begin{tabular}{|ll|}
\hline Question & Answer \\
\hline Will follow-on funding (post-LDRD project) be applied for? & Yes \\
\hline Source of support for follow-on funding? & GCEP \\
\hline Has follow-on funding been obtained? & Yes \\
\hline Amount of follow-on funding (\$K) & $\$ 1.77 \mathrm{M}$ \\
\hline Number of Post Docs supported by LDRD project? & 1 \\
\hline Number of students supported by LDRD project? & 0 \\
\hline Number of scientific staff/technical staff hired with LDRD funding? & 0 \\
\hline Number of copyrights filed (beyond publications)? & 0 \\
\hline Number of invention disclosures filed? & 0 \\
\hline Number of patent applications filed? & 0
\end{tabular}




\subsection{Correlated electron physics at oxide interfaces}

Principal Investigator: Srinivas Raghu

\section{Project Description}

A pioneering set of experiments involving the interface between $\mathrm{LaAlO} 3$ and $\mathrm{SrTiO} 3$ (both of which are insulators), have revealed that high mobility metallic behavior, even superconductivity, occurs as the voltage across the interface is increased. These observations are certainly of fundamental importance as they point towards a new arena of designing novel phases of matter at interfaces between distinct, highly correlated electron materials. They also have tremendous scope for energy and materials applications in the next generation of devices such as field effect transistors that are based on correlated oxides instead of semiconductors. Transport measurements in these systems suggest that superconductivity occurs when there are sizeable spin-orbit interactions. Even ferromagnetism has been observed at the interface and is thought to coexist with superconductivity. Since ferromagnetism is typically a phenomenon associated with strong electron-electron interactions, it is clear that the oxide interface experiments pose a rich set of problems involving the interplay between electron correlations, spin-orbit coupling and the reduced dimensionality associated with the interface.

This project aims to establish a new direction within SLAC, wherein fundamental theoretical calculations involving a variety of analytical and computational methods will be performed to understand the basic phase diagram of these systems and to predict phenomena at interfaces between other transition metal oxides.

\section{Accomplishments}

I have developed a theory of magnetism at the interface between $\mathrm{LaAlO} 3$ and SrTiO3. In collaboration with physicists at Cornell (Eun-Ah Kim and Mark Fischer) we have developed a theory of magnetism from the perspective of a two dimensional electron gas with Rashba spinorbit coupling at light densities. Our results were presented at the American Physical Society March Meeting in Boston, MA. Our paper has been published in the Physical Review.

I have written a paper with Ted Geballe on the role of screening in the transition temperature of an unconventional superconductor. We suggest that the disparity in Tc among the cuprate superconductors can be attributed to charge reservoir layers (CRLs) that are capacitively coupled to electrons in metallic layers of the cuprate materials that participate in superconductivity. By screening longer range coulomb interactions, the CRLs play a significant role in determining the optimal transition temperature of these systems. The CRLs, coupled capacitively with electrons in the copper-oxide plane form natural "oxide interfaces" that we have studied in various theoretical limits. Our studies suggest new strategies for engineering a high temperature superconductor using methods that scientists studying oxide interfaces have been developing in recent years. Our paper is published in Physical Review B and has so far been well-cited. 
Recently, I have completed a paper in collaboration with Harold Hwang's group on interfacial superconductivity in a novel bilayer system. The paper has been accepted as a rapid communication in PRB.

I am in the final stages of writing a review paper titled "Frontiers of Interfacial Superconductivity" with Chris Bell and Harold Hwang.

\section{Publications}

C. Bell, S. Raghu, and H.Y. Hwang, "Frontiers of interfacial superconductivity", Review for Reports of Progress in Physics (RPP), Manuscript in preparation.

\section{Questionnaire}

\section{Question}

Answer

Will follow-on funding (post-LDRD project) be applied for?

Yes

Source of support for follow-on funding?

DOE(SIMES)

Has follow-on funding been obtained?

Yes

Amount of follow-on funding (\$K)?

150

Number of Post Docs supported by LDRD project?

0

Number of students supported by LDRD project?

2

Number of scientific staff/technical staff hired with LDRD funding?

0

Number of copyrights filed (beyond publications)?

0

Number of invention disclosures filed?

0

Number of patent applications filed?

0 


\subsection{Non Fermi Liquids}

Principal Investigator: Srinivas Raghu

\section{Project Description}

The goal of this project is to foster collaborations among theorists in Photon Science and PPA to investigate quantum field theories of strongly correlated systems. The theorists involved will use powerful methods of effective field theory to obtain new solvable limits and qualitative insight into the nature of strongly correlated materials, especially non-Fermi liquid metals Is superconductivity enhanced in a metal near a quantum critical point? Can higher $T_{c}$ superconductivity be realized in a metal with ill-defined quasiparticles? These questions are inspired by some of the materials of greatest interest in condensed matter physics, which continue to defy theoretical understanding. These include, for instance, the high temperature superconductors and heavy fermion systems. While the ground states of these materials are conventional, the higher temperature phases are strongly coupled phases of matter. For instance, high temperature superconductivity in cuprate and iron pnictide materials develops out of a metallic phase that bears little resemblance to a free electron model with small attractive interactions. This is visible from basic properties of this phase, such as its resistance to the flow of electrical currents. Controlled theoretical models, which reproduce all the behaviors of this phase are still lacking.

Our goal over a three-year period is to design a wider class of controlled models exhibiting nonFermi liquid phases, to understand their instabilities to conventional ground states such as superconductivity, and to work toward more realistic models without losing our control of the calculations. Specifically, in the first year, we will initiate work on developing field-theoretic models of controlled fixed points that could emerge from interactions of fermions at a Fermi surface with additional bosonic fields (surprisingly, still a problem that remains largely open in 2 spatial dimensions, the dimension of relevance to the cuprate superconductors); and we will improve some of the most unrealistic features of the holographic models of non-Fermi liquids (by, for instance, relaxing the large $\mathrm{N}$ approximation, perhaps by using large density as a parameter instead).

In the longer term, our goal is really to improve understanding of the materials that exhibit nonFermi liquid behaviors. A wide variety of experiments give hints of power law behaviors in e.g. specific heat and resistivity measurements in various metals, which differ from those of the conventional Fermi liquid. The "non-Fermi liquids" presumably comprise a wide class of rather different possible behaviors of interacting electrons, none of which are well understood at present. We are especially interested in predicting spectral properties of non-Fermi liquid materials, which are routinely studied by experimentalists at SIMES. Development of even quasirealistic, controlled models of such phases would justify our effort. But a more optimistic, long term outcome of this effort, in a scenario where the project were extended beyond an era of LDRD seed funding by continued BES and/or HEP support, would be a theory which is sufficiently well- 
developed to explain specific behaviors in certain classes of materials, and to guide investigation of new promising materials.

In the future, this project will involve collaboration with computational scientists. We will investigate the entanglement properties of non-Fermi liquids using Density Matrix Renormalization Group (DMRG) simulations, and will collaborate with Hong-Chen Jiang, an exceptional staff scientist in Photon Science working on computational aspects of condensed matter physics.

\section{Accomplishments}

The LDRD project led to the publications listed below.

\section{Publications}

A.L. Fitzpatrick, S. Kachru, J. Kaplan, S. Raghu, G. Torroba, H. Wang, “Enhanced Pairing of Quantum Critical Metals near d=3+1", arXiv:1410.6814.

A. L. Fitzpatrick, S. Kachru, J. Kaplan, S. Raghu, "Non-Fermi Liquid Behavior of large NB Quantum Critical Metals", Phys. Rev. B 89, 165114 (2014).

H. Watanabe, S. Parameswaran, S. Raghu, A. Vishwanath, "Anomalous Fermi Liquid Phase in Metallic Skyrmion Crystals", Phys. Rev. B 90, 045145 (2014).

\section{Workshops}

None

\section{Questionnaire}

Will follow-on funding (post-LDRD project) be applied for?

Yes

Source of support for follow-on funding?

DOE

Has follow-on funding been obtained?

$\mathrm{NO}$

Amount of follow-on funding $(\$ \mathrm{~K})$ ?

Number of Post Docs supported by LDRD project?

Number of students supported by LDRD project?

Number of scientific staff/technical staff hired with LDRD funding?

0

Number of copyrights filed (beyond publications)?

Number of invention disclosures filed? 
Notes on number of post-docs/students/staff: any post-doc or student who was supported for more than $10 \%$ of his/her time should be included here. For staff, this is for staff that were hired specifically for and funded by the LDRD project (at least $50 \%$ of their time). 


\subsection{Prototype for a Microjoule-class Femtosecond XUV Source}

Principal Investigator: David A. Reis

\section{Project Description}

Conventional coherent XUV light sources are based on high---order harmonic generation (HHG) in gases. In a typical setup, the maximum pulse energy is in the nanojoule level. Much brighter XUV pulses are desired for many applications including ultrafast time---resolved photoemission, coherent imaging, lithography, and to externally "seed" future soft---x---ray free---electron lasers. The high---density solids are potentially more efficient interaction media but the bulk samples get multi---shot sample damage and the XUV photons get re---absorbed in the sample as the photon energy is well above the band---edge. In this LDRD project, we are testing a new HHG scheme, where the target is crystalline nanoparticle spray - a jet of flowing nanoparticles. This scheme is virtually immune to effects of material damage at high intensity as the target is being constantly replenished and the effective density is expected to be $\sim 100$ times atomic density of typical gas target, but with a minimal self---absorption effects. Final goal is to reach the microjoule---level of pulse energy in the $20-50 \mathrm{eV}$ photon energy range in a tabletop setup.

\section{Accomplishments}

This LDRD has considered the evaluation of a new approach of HHG in a novel active medium containing a continuous flow of crystalline nanoparticles. This is a potential alternative approach to the conventional gas phase HHG, where the efficiency is low. In the gas phase, in the XUV photon energy range $(20 \mathrm{eV}$ to $50 \mathrm{eV})$ efficiency is in the range from $10^{---6}$ to $10^{---8}$

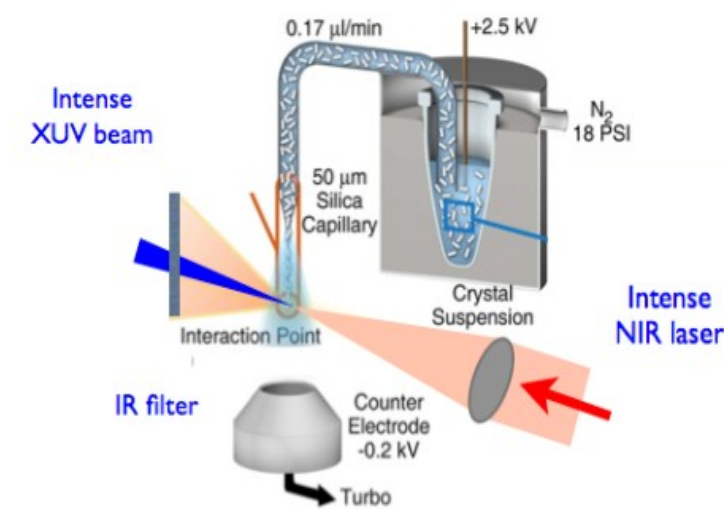

Figure 1: Schematics of the concept of the XUV source on crystalline nanoparticle spray. Intense NIR laser beam intercepts a nano-droplet jet that has flowing solid nanoparticles. The nonlinear interaction generates XUV pulse that can be separated from the residual NIR laser pulse. such that the output is at the nanojoule level, for a typical milijoule---class driving pumps. One way to increase the efficiency would be by increasing the gas pressure at the target but above a certain limit ( $\sim 50$ Torr for Ar gas at $\sim 800 \mathrm{~nm}$ ) that would not be a viable option because of the phase--- matching limitations (and depletion). Another way would be what is known as the brute force method i.e. by using substantially large driving pumps (Joule--class) and large focusing cross---sections, at fairly low repetition rate (limited by the currently available laser technology). Our scheme has a small footprint ( $<2$ meter) and currently operates at $1 \mathrm{kHz}$ repetition rate on a commercial Ti:sapphire laser system. The schematics of the light source is shown in figure 1, where strong Ti:sapphire laser pulses intercept the crystalline nanoparticle spray such that every laser pulse 
interacts at the replenished interaction spot and the intense XUV pulses are filtered through ultra---thin metal filter. After the filter XUV beam is dispersed by using a diffraction grating.

Demonstration of a working scheme: In the first year, we have successfully tested our new scheme, which included measurement of the spectrum in the photon energy range from about $15 \mathrm{eV}$ to $50 \mathrm{eV}$. We used $\mathrm{MgO}$ nano---particles suspended in a highly charged liquid buffer to make nano---droplets jet using the electrospray method. This jet intercepts the focal spot of a milijoule---class femtosecond Ti:sapphire laser beam of wavelength $800 \mathrm{~nm}$ as shown in figure 1 . Thus, it creates a virtually damage free sample environment since as the target gets constantly replenished for consecutive laser shots with peak intensity on order of $10^{14} \mathrm{~W} / \mathrm{cm}^{2}$. Strong---field response in crystalline nanoparticles is expected to produce high order harmonics. Because of the sub---micron scales of the individual nano---droplets self--absorption of high---energy photons in the interacting medium is substantially reduced compared to the case of fixed bulk target. The figure 2 shows a measured XUV spectrum where the red arrows represent the expected location for various harmonic orders. These test results complete an important milestone of the project-----demonstration of a working scheme.

XUV Efficiency in active medium: We have compared the generation efficiency in this light source to the gas phase HHG (Ar gas target) using the same spectrometer. For the same input pulse energy $(1 \mathrm{~mJ})$ the total yield in this detection range was significantly higher in the case of electrospray target. The measured XUV spectrum is shown in figure 2.

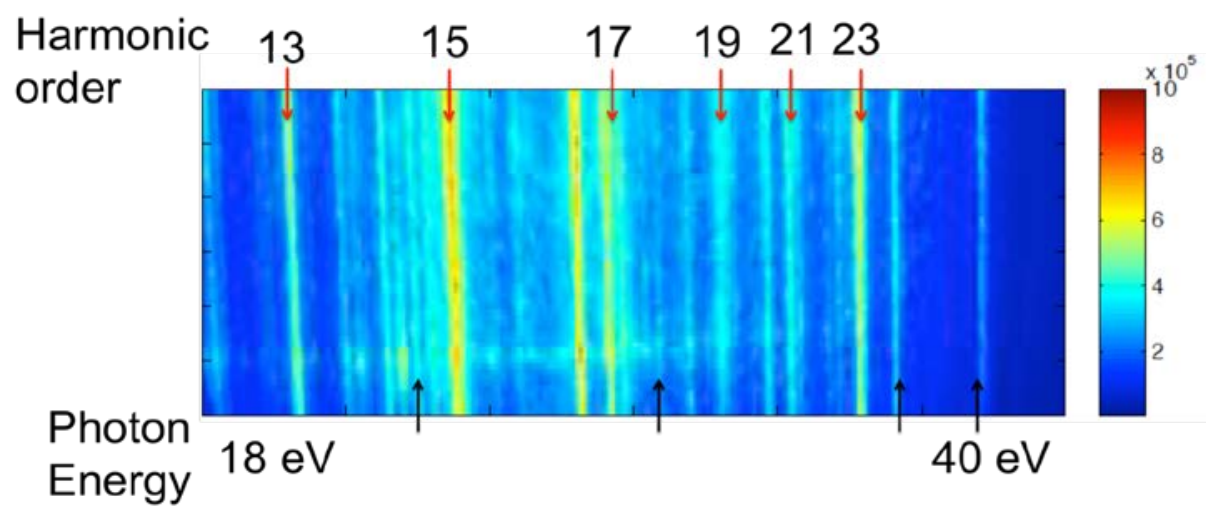

Figure 2: Measured spectrum of the XUV harmonic source under construction with this LDRD. Red arrows are expected locations of harmonics along with their respective orders and black arrows are (some of) the incoherent plasma lines as characterized by their narrow line---widths.

Characterization in--progress: The experiments to measure coherence properties of the XUV are in progress. This includes the Young's double slit experiment. Measurement of spatial coherence is important because, as seen in figure 2, in addition to the harmonic lines we have also observed other spectral lines as (some of them marked by the black arrows). These lines are likely the plasma lines, as characterized by their narrow bandwidth features. 
Second year: We plan to optimize the harmonic yield with the size and type of particle and measure the polarization properties and the pulse---to---pulse stability of the output, which is consistent to the outlined milestones in this two year project. Depending on the results of the coherence measurements (content of the plasma emission from residual liquid), we may need to consider simplifying the particle delivery method, for example, "all---solid" active medium. The laser system will be upgraded to achieve higher driving pulses energies at 800 $\mathrm{nm}$.

\section{Outcome (e.g. Publications)}

New FWP: our progress in the first year of the LDRD helped to strengthen Ghimire Early Career proposal, which is a new FWP now.

High---repetition rate Initiatives: Success on this LDRD project prepares us for developing high repetition rate $(\sim 100 \mathrm{kHz})$ harmonic source that would bring unique capabilities to SLAC particularly to match the specifications of LCLS---II. This prototype also exceeds the requirements as an external "seed" for a soft x---ray FEL when combined with EEHG /HGHG scheme and could be relevant to R \& D in this field.

\section{Publications}

None

\section{Workshops}

None

\section{Questionnaire}

\section{Question}

Will follow-on funding (post-LDRD project) be applied for?

Source of support for follow-on funding?

Has follow-on funding been obtained?

Amount of follow-on funding $(\$ \mathrm{~K})$ ?

Number of Post Docs supported by LDRD project?

Number of students supported by LDRD project?

Number of scientific staff/technical staff hired with LDRD funding?

Number of copyrights filed (beyond publications)?

Number of invention disclosures filed?

Number of patent applications filed?

\section{Answer}

Yes

DOE, DOD

Yes

$2.5 \mathrm{M}$

0

0

0

0

0

0 


\subsection{Towards the SLAC Femtosecond Macromolecular Micro and Nanocrystallography Facilities}

Principal Investigator: Mike Soltis

\section{Project Description}

Development of an innovative approach to the very accurate, high resolution structure determination of complex, radiation sensitive biomolecular complexes and reaction intermediates in their catalytic cycles using femtosecond LCLS x-rays and micro-to-nano crystals. Application to energy relevant problems, i.e. hydrogenase (which catalyzes the bio-production of hydrogen) and polyketide synthase (a bio-assembly line for green chemistry), drives the development of an integrated platform for streamlining and optimizing an experimental approach for related projects and the general user community, focused on sample preparation, handling and characterization, sample delivery, data collection and data analysis.

\section{Accomplishments ( $3^{\text {rd }}$ Year)}

Activities in year 3 focused on new sample characterization developments, new delivery devices and methods and data collection strategies using the goniometer setup at the LCLS XPP station.

Nanocrystal Characterization: A
systematic protocol was
developed to characterize
"nanocrystals" (<5 $\mu \mathrm{m}$ in size)
using light microscopy, light
scattering and electron
microscopy (EM) in collaboration
with G. Calero (University of
Pittsburgh). UV tryptophan
fluoresce microscopy was used
to initially identify proteins in
crystallization drops. Drops
yielding a fluorescence signal
were then characterized with

EM. UV-negative samples and clear drops were further analyzed using standard Dynamic Light

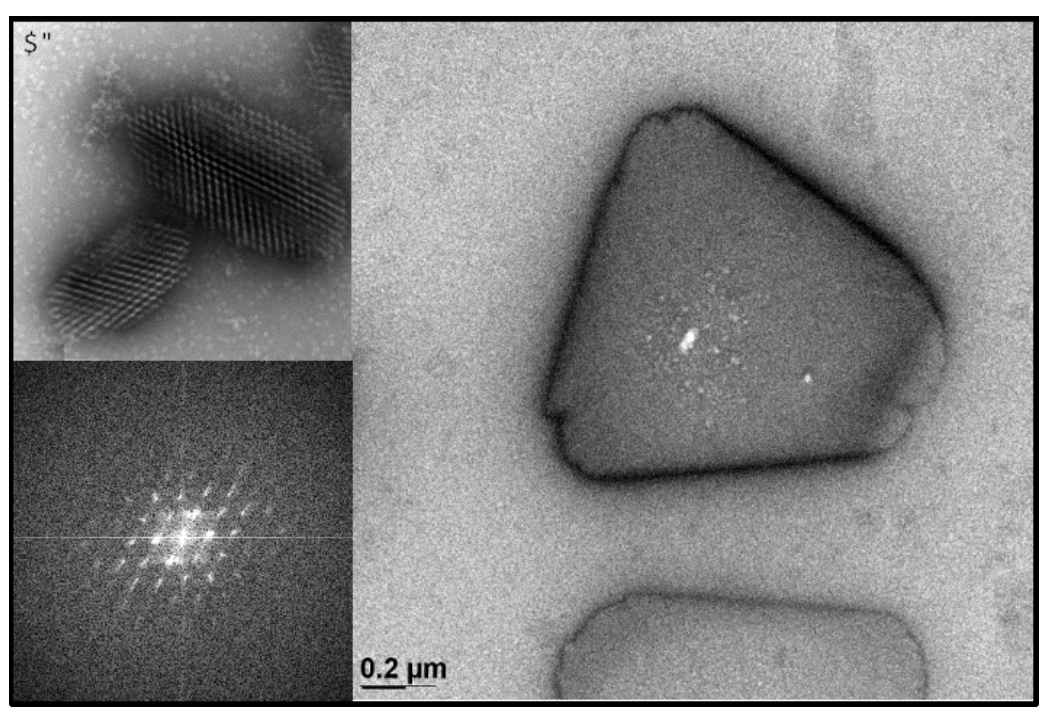

Figure 1: Electron microscopy image (right) and accompanying lattice (upper left) and reduced FFT (lower left) of the megasynthesis polyketide DEBS complex.

Scattering (DLS) techniques,

which can detect particles with 50-1000 nm radii. For solutions containing crystals, EM was also used for further characterization.

For most samples tested, EM visualization (Fig. 1) provided an efficient method to differentiate between nanocrystals and large protein aggregates that were detected by the UV-microscopy or DLS methods. Since EM allows visualization of crystal lattices, protein nanocrystals could be 
discriminated from salt crystals, which were frequently coated with protein aggregates, yielding a false-positive UV-signal. Detection of protein nanocrystals was possible for the majority of samples tested. Moreover, the highly ordered crystal lattice observed for nanocrystals of the polyketide DEBS complex (6-deoxyerythronolide B synthase) correlated with the high resolution diffraction observed during experiments on LCLS CXI (resolution observed to $1.8 \AA$ ). Based on these and other results, guidelines will be developed for general characterization of nanocrystals in solution using these approaches.

EM was also used to characterize crystals post-injection using nozzles from the most commonly used injector (GDVN). It was found that in some cases the diffraction quality of the nanocrystals, particularly those with high solvent content, changed after the delivery process, and that the crystal lattice was visibly damaged after injection.

High-speed Imaging for Monitoring Sample Delivery: A new high-speed imaging setup using 10x and 20x long-working distance objectives was developed in the Photon Science group at SLAC and became a permanent part of the CXI endstation at LCLS. The optical setup can resolve features as small as $800 \mathrm{~nm}$, and can be used in two modes: 1) Time-resolved imaging with femtosecond illumination, producing one image per LCLS pulse. This mode of operation is available for CXI experiments where other instrumentation does not block the illumination path, and can have a timing resolution down to 1 ps; and 2) Imaging with a user-supplied high-speed camera. A white-light illumination setup provides enough light for cameras that operate up to 2 million frames per second. The time-resolved mode of imaging was used on CXI to record XFELinduced explosions of the jet and drops at equivalent frame rates up to 200 million fps. Preliminary analysis indicates that the GDVN jet can be used at the full rate of the European XFEL. The high-speed mode of operation was used to monitor sample delivery with a new LCP "toothpaste" jet, at imaging rates up to 100,000 fps. High-speed monitoring improved the optimization of the jet parameters, and it was also discovered that at moderate pulse powers, the LCP jet quickly self-heals after a hole is blown by a pulse.

Alternative Sample Delivery Systems for XFEL and Synchrotron Experiments: Because the GDVN liquid jet injector, which has been used for the majority of XFEL macromolecular crystallography studies to date, has an intrinsically low hit rate, the majority of crystals are "wasted" during a typical diffraction experiment. There is also evidence that some crystals may suffer during the delivery process as described above. Thus, alternate data collection methods have also been pursued that maximize hit rate and minimize sample waste.

Electrospinning Jet: An alternative liquid injector was developed by the Photon Science group at SLAC based on electro-spinning principles. The jet uses a charge differential and modest pressure flow. The flow rate is reduced by up to a factor of 30 compared to the GDVN, greatly reducing the amount of material consumed. The system was first tested on SSRL BL12-2 and then used for data collection on the LCLS CXI station. It is now offered to general users as an alternative to the GDVN injector. A SLAC and the Stanford Institute for Chemistry, Engineering \& Medicine for Human Health (ChEM-H) Seed Grant entitled “Understanding Antibiotic Resistance: TimeResolved XFEL Structural Studies of Wild-type and Mutant Ribosomes" involving SMB SSRL and 
Photon Science staff was awarded in 2013 (Soltis and Puglisi, PIs) that will use a modified version of the electro-spinning liquid jet for kinetic studies of drug interactions with ribosomes.

Microfluidic Droplets for Sample Delivery: Compared to a continuous jet, the microfluidic droplet approach has advantages of very low sample consumption, high hit rate, insensitivity to the properties of the crystal liquor, and the straightforward extension to advanced drop manipulations (sorting, mixing, optical pumping) already demonstrated with on-chip microfluidic devices. A prototype was designed in the Photon Science group at SLAC with a coaxial flow system made from an outer $360 \mu \mathrm{m}$ capillary and an inner $150 \mu \mathrm{m}$ capillary. The outer capillary supplies a volatile oil while the inner capillary supplies the sample of interest. The droplets are pulled periodically from the inner capillary using high-voltage pulses applied to an electrode. To reduce velocity dispersion as the drops travel in a tube much larger than their diameter, the droplets enter a $50 \mu \mathrm{m}$ ID capillary for transport through the dispensing jet. Work is underway to test the stability of drop generation when using drops loaded with crystals, and to ruggedize the setup for routine experimental use. Extracting the drops from the capillary into vacuum while synchronized with LCLS pulses, remains a challenge.

Goniometer-Based Data Collection: Data were collected from standard loops and meshes using newly developed raster and helical data collection routines. In some cases, high capacity polyethylene sample grids were used (Fig. 2), which are compatible with the SAM robot and can hold up to 70 frozen crystals each. Each crystal can be automatically translated and exposed for highly efficient data collection.

Data were collected from a multitude of metalloprotein complexes: myoglobin (Cohen, SSRL), FeFe hydrogenase (Peters, Montana State), cyclophilin A (Fraser, UC San Francisco), photosystem II (Kern, LBNL), homoprotocatechuate 2,3-dioxygenase (Lipscomb, University of Minnesota), methane (Lipscomb, University of Minnesota), bacterial dyedecolorizing peroxidase (Lipscomb, University of Minnesota), cytochrome c peroxidase compound I (Poulos, UC Irvine) and cytochrome ba3 oxidase (Stout, The Scripps Research Institute). The "still" diffraction images were processed with new software recently developed in the Sauter (LBNL) and Brunger (Stanford) labs particularly optimized for XFEL data.

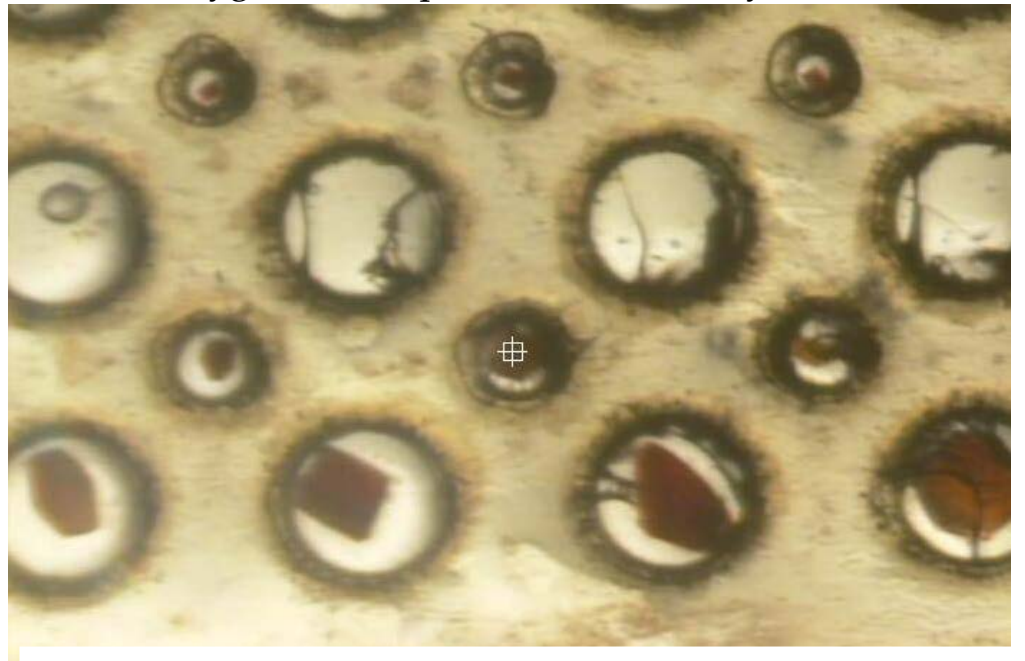

Figure 2: Newly designed grid for mounting and static delivery of crystals to the LCLS beam. Myoglobin crystals (red) are shown mounted in matching-size holes. 
The metalloprotein structures were solved by molecular replacement and the resulting electron density maps were of excellent quality (myoglobin heme site shown in Fig. 3). Crystals from several systems were also screened and several showed improved resolution and diffraction quality compared to synchrotron data: GCPR (Kobilka \& Weis, Stanford University), T4 base plate (Rossman, Purdue University), N-ethylmaleimidesensitive factor (Brunger, Stanford University), HIV-1 env trimer (Wilson, The Scripps Research Institute) and HIV gp120 (Harrison,

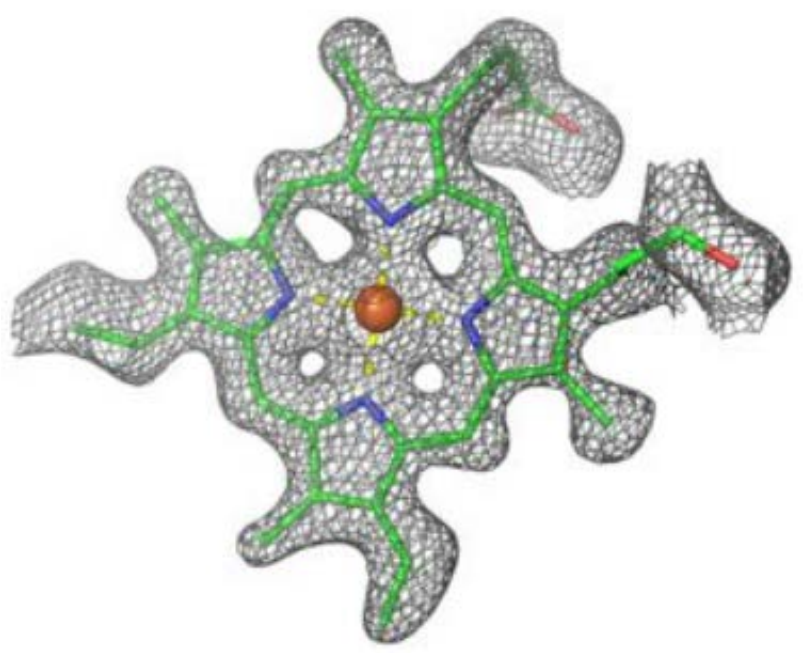
Harvard). The diffraction resolution for the GPCR crystals tested was extended by $0.6 \AA$, to $2.3 \AA$ compared to $2.9 \AA$ recorded at APS GMCA. These results along with those obtained with data recorded at CXI, have generated much interest by the community and the funding agencies. As a result, and with the initial efforts in part though this LDRD, and in strong collaboration and further developments with LCLS staff, SLAC has recently obtained funding from Federal sources (DOE BER, NIH NIGMS, DOE BES) and Stanford University to build a dedicated goniometer-based station at LCLS. This new Macromolecular Femtosecond Crystallography (MFX) station will be developed and implemented by the LCLS in collaboration with the SMB group at SSRL.

Summary: The results from the LDRD developments have shown that nanocrystal solutions can be properly prepared and characterized for quality diffraction experiments using the XFEL and that high quality "radiation damage free" macromolecular structures can be obtained efficiently using a minimal amount of sample. These developments contributed to the building of a new macromolecular crystallography end station (MFX), which was one of the main goals of this LDRD project. 


\section{Questionnaire}

\begin{tabular}{|ll}
\hline Question & Answer \\
\hline Will follow-on funding (post-LDRD project) be applied for? & Yes \\
\hline Source of support for follow-on funding? & NIH \\
& DOE BER \\
& Stanford ChEM-H \\
& Institute \\
\hline Has follow-on funding been obtained? & \\
\hline Amount of follow-on funding (\$K)? & Yes \\
\hline Number of Post Docs supported by LDRD project? & $\sim 5,800$ (MFX) \\
\hline Number of students supported by LDRD project? & 1 \\
\hline Number of scientific staff/technical staff hired with LDRD funding? & 0.5 \\
\hline Number of copyrights filed (beyond publications)? & 0 \\
\hline Number of invention disclosures filed? & 0 \\
\hline Number of patent applications filed? & 0 \\
\hline
\end{tabular}




\subsection{Modeling Conformational Ensembles of Proteins and Complexes}

Principal Investigator: Henry van den Bedem

\section{Project Description}

Macromolecules often exhibit significant conformational flexibility, especially when they interact with ligands or protein partners to form assemblies. Probing these conformational changes is essential to understanding their cellular function, yet these scientifically important molecules are often difficult to crystallize and diffract poorly. We started a computational research program to model challenging systems with experimental data from multiple sources, including protein crystallography, small angle X-ray scattering and nano-crystallography. Our approach is to extend an innovative, robotics-inspired Kino-Geometric Sampler conformational search algorithm (KGS) to fit previously determined domains or proteins to experimental data. KGS exhibits a singularly large radius of convergence and optimally reduces the number of free parameters. These unique features enable flexible 'docking' of atomic models in low-resolution data while moderating the risk of overfitting. We aim to automatically compute 3-D models, and to provide structural insights for systems that diffract poorly or cannot be crystallized. Our method will also provide insight into functionally relevant dynamics, especially for time-resolved experiments or a series of static structures

\section{Accomplishments}

A Research Assistant was hired starting Jan 12012 to implement a gradient descent protocol in KGS to guide conformational variability along experimental data. A post-doctoral scholar, Dr. Dimitar Pachov joined the project on Feb 1 2012. The gradient of the data is projected onto the null-space of the first-order approximation to the kinematic model, i.e. $\mathrm{d} q=N N^{T} \nabla \rho$, where $q$ is the current conformation, $N$ is a basis for the null space, and $\rho$ is experimental data. A gradient descent is then successively applied to rigid bodies. Tests on simulated data corresponding to the closed conformation of LAO protein (PDB ID 2LAO) revealed that KGS converged to a good data fit starting from the open conformation within no more than 20-30 gradient descent cycles, spanning a radius of convergence exceeding $4.5 \AA$. This exciting result affirms that null-space optimization maintains the performance of KGS even with a vast number of kinematic cycles, a key year 1 research objective. It reinforces the potential of KGS for efficient modeling of structural assemblies into low resolution data.

A collaboration with Dr. Julie Bernauer at INRIA/AMIB in Paris, France was initiated to extend our modeling approach to RNA and protein/RNA complexes. Supported by INRIA, Rasmus Fonseca joined the project in FY13 as a post-doctoral scholar. Dr. Fonseca visited SLAC Jan 15 May 23, 2013 to develop methods and algorithms for KGS.

In FY13 we set the goal to extend KGS to model conformational flexibility of complexes. A challenge is to maintain inter-molecular hydrogen bonds while sampling conformational 
variability. We derived a mathematical model to extend the kinematic model to multiple molecules by adding global degrees of freedom. The global degrees of freedom account for rigid body motions of the molecules, and help maintain inter-molecular hydrogen bonds. Tests on small systems show promising results, but bond lengths still show too much variation despite carefully calibrated numerical step sizes.

In FY13 we started preparing two manuscripts. The first manuscript presents the computational extension to complexes and density fitting. It describes the application of these new capabilities to the GPCR 32 AR:Gs complex. The second manuscript presents extension of the kinematic model to RNA molecules. We furthermore show that the calculated chemical shifts of the sampled conformational states of RNA agree with experimental chemical shifts. Thus, the sampled states approximate the ensemble in solution.

In FY14 we focused on calibrating our algorithms and applying our models and procedures to biomedically relevant systems. The results of our research were disseminated through 3 peerreviewed publications in high-impact factor journals this year, with several more to be submitted within the next few weeks. We have made the software available for download. We are collaborating with professor James Bardwell (HHMI/U Michigan), and have started collaborations with professor Dan Herschlag and professor Bill Weis (both Stanford) to use our procedures for interpreting their data collected at SLAC.

In FY14, together with professors Levitt and Kobilka (Stanford) we submitted a grant application to NIH for follow-on funding. While we were unable to secure funding in the first round, we will resubmit the proposal in Feb 2015.

\section{Publications}

Budday, D., Leyendecker, S., \& van den Bedem. H. "Rigidity and floppy modes in non-generic protein configurations". (In preparation)

Pachov, D. P., Fonseca, R, Arnol, D. ,Bernauer, J. \& van den Bedem, H. “Conformational coupling in ensembles of inactive and activated $\beta 2 A R: G \alpha s "$. (In preparation)

Pachov, D. \& van den Bedem, H. "Nullspace sampling with holonomic constraints reveals molecular mechanisms of protein Gas." PLOS Comput. Biol. In review (2014)

van den Bedem, H. \& Fraser, J.S. "Integrative, Dynamic Structural Biology at Atomic Resolution-It's About Time". Nat Meth. In review (2014)

Fonseca, R, Pachov, D. P., Bernauer, J. \& van den Bedem, H. "Characterizing RNA ensembles from NMR data with kinematic models". Nucl. Acids Res. 42:9562-9572 (2014)

van den Bedem, H., Bhabha, G., Yang, K., Wright, P. E. \& Fraser, J. S. "Automated identification of functional dynamic contact networks from X-ray crystallography". Nat Meth 10, 896-902 (2013).

\section{Workshops}

"Characterizing Biomolecular Ensembles with Molecular-Scale Kinematics". Department of Computer Science, Rice University, Sep 2014. 
"Integrative dynamic structural biology at the atomic scale-It's about time". Department of Chemistry, Utrecht University, The Netherlands, Jul 2014.

"Molecular-Scale Kinematics in Computational Structural Biology". Department of Mechanical Engineering, University of Erlangen-Nuremberg, Germany, Jun 2014.

"Now You See Me, Now You Don't: Structurally Characterizing Biomolecular Dynamics from Kinematics". Institute for Computational and Mathematical Engineering. Stanford University, May 2014.

"Now You See Me, Now You Don't: Structurally Characterizing Biomolecular Dynamics from Kinematics". Department of Computer Science, Duke University, May 2014.

H. van den Bedem, "Identifying functional dynamic networks from X-ray data". Integrative Approaches for Modeling Biomolecular Complexes. Nice, France. May 2013.

H. van den Bedem, "Identifying functional dynamic networks from protein X-ray data". International Conference for Biomolecular Dynamics: Experiment Meets Computation. King Abdullah University of Science and Technology, KSA. Feb 2013.

H. van den Bedem, "Back to the Future: Multi-Conformer Contact Networks in Room Temperature PX Data Elucidate NMR Conformational Dynamics". 70 ${ }^{\text {th }}$ Annual Pittsburgh Diffraction Conference. Stanford, CA. Oct 2012

H. van den Bedem, "Multi-Conformer Contact Network Analysis Provides The Structural And Functional Basis For NMR Relaxation Experiments", Computational Methods in Structural Biology, Workshop, Hong Kong University of Science and Technology, Aug 1-3, 2012. Invited.

H. van den Bedem, "Multi-Conformer Contact Network Analysis Provides The Structural And Functional Basis For NMR Relaxation Experiments", 3DSig 2012 Structural Bioinformatics and Computational Biophysics, Long Beach, CA, Jul 13-14, 2012. 


\section{Questionnaire}

\begin{tabular}{ll}
\hline Question & Answer \\
\hline Will follow-on funding (post-LDRD project) be applied for? & Y \\
\hline Source of support for follow-on funding? & NIH \\
\hline Has follow-on funding been obtained? & N \\
\hline Amount of follow-on funding (\$K)? & 150 \\
\hline Number of Post Docs supported by LDRD project? & 1 \\
\hline Number of students supported by LDRD project? & 0 \\
\hline Number of scientific staff/technical staff hired with LDRD funding? & 0 \\
\hline Number of copyrights filed (beyond publications)? & 0 \\
\hline Number of invention disclosures filed? & 0 \\
\hline Number of patent applications filed? & 0
\end{tabular}




\subsection{Methodology Developments for Structural Studies of Post- translational Modifications of Proteins}

Principal Investigator: Soichi Wakatsuki

\section{Project Description}

Post-translational modification of proteins, such as ubiquitination, glycosylation, phosphorylation and acetylation, play critical roles in many cellular events and functions, such as cell cycle control, quality control, and stem cell development. In this LDRD project, we aim to continue to develop new methodologies to discover novel functions of polyubiquitin chains and other post-translational modifications of proteins. We will further establish a research environment for protein production, purification, crystallization and biochemical and biophysical characterization. Using this infrastructure, we will enzymatically produce polyubiquitin chains of different linkages and lengths. The produced polyubiquitin chains will be used to screen protein complexes in various organisms to find new polyubiquitin signaling pathways. Novel protein complexes will be subjected to functional and structural studies using synchrotron protein crystallography and solution scattering and radiation-damage free XFEL nano-crystallography.

\section{Accomplishments}

Establishing infrastructure and collaborations: During FY14, we expanded further the infrastructure for protein expression and purification, and characterization of protein complexes involved in posttranslational modifications, in particular protein ubiquitination and ubiquitin chain recognition. After an extensive survey of state-of-the-art protein crystallization robots, a system was procured and has produced number of protein crystals which were used for structure determination. A new collaboration was established with Prof. Daria Mochly-Rosen of Stanford School of Medicine on drug discovery project on an enzyme and a seed funding was secured from Child Health Research Institute of Stanford School of Medicine, a two-year grant, \$23,250 for FY13-14. Another collaboration project was formed with Prof. Dave Stahl, University of Washington, a BERAC member, on ammonia oxidation, a key step in nitrogen cycle. Also discussions on collaboration projects with EMSL/PNNL have been initiated.

Development of a new LCLS beamline, Macromolecular Femtosecond Crystallography: We initiated a new project to develop and construct a dedicated atmospheric pressure Macromolecular Femtosecond Crystallography (MFX) instrument installed inside a new experimental hutch, referred to as Hutch 4.5, on a new hard X-ray beamline in at LCLS. Multiple crystal delivery systems and approaches will be accommodated by MFX, such as robotic fixedtarget goniometer systems, on-demand liquid sample delivery systems, and LCP (lipidic cubic phase) injectors for membrane proteins. The system will also be highly flexible to allow integration of potential new sample delivery techniques to be developed. The development will be leveraged by the experiences and expertise accumulated at the CXI and XPP stations by the consortium members. Initial investments have been obtained from DOE-BER (\$2M, Wakatsuki as 
PI), NIGMS (\$1M, Wakatsuki as PI), and LCLS and DOE-BES. The project was launched in April 2014. Further funding from federal agencies and private foundations is being explored.

Development of a new phasing method using XFEL: Utilization of two-color XFEL pulses for de novo structure determination was further defined and developed, and a 5-day experiment LD91 was conducted on LCLS CXI station. Methods for achieving further energy separation were identified and pursued. A successful LD91 beam time at CXI on June 13 to 17, 2014 followed, where LCLS was set up to run in a two-color self-seeded mode. The lower energy pulse was placed at the inflection point for the $\mathrm{Yb} \mathrm{L3}$ edge at $8947 \mathrm{eV}$ and the higher energy pulse $95 \mathrm{eV}$ above that to allow for the MAD phasing using ytterbium as the anomalous scatterer. The two pulses were 10-15 fs long and almost coincidental in time with about $15 \mathrm{fs}$ time difference. They were about equal in average strengths and most pulses contained most of the energy in the peaks with only a small fraction in the SASE background. These doubly-seeded two-color pulses produced diffraction patters with doublets corresponding to the two colors with anomalous signals. High resolution anomalous maps were produced from the diffraction images of lysozyme micro crystals. New algorithms for full exploitation of the two-color mode are being developed. Several new LCLS beamtime proposals were submitted based on these results. 


\section{Publications}

Hiraki M, Yamada Y, Chavas LM, Wakatsuki S, Matsugaki N.J. ,"Improvement of an automated protein crystal exchange system PAM for high-throughput data collection", Synchrotron Radiat. 2013 Nov;20(Pt 6):890-3

Yamada Y, Chavas LM, Igarashi N, Hiraki M, Wakatsuki S, Matsugaki N. J "Improvements toward highly accurate diffraction experiments at the macromolecular micro-crystallography beamline BL-17A." Synchrotron Radiat. 2013, 20, 938-42

Suzuki H, Tabata K, Morita E, Kawasaki M, Kato R, Dobson RC, Yoshimori T, Wakatsuki S. "Structural basis of the autophagy-related LC3/Atg13 LIR complex: recognition and interaction mechanism", Structure. 2014 Jan 7;22(1):47-58. doi: 10.1016/j.str.2013.09.023.

Fujita H, Rahighi S, Akita M, Kato R, Sasaki Y, Wakatsuki S, Iwai K. “Mechanism Underlying I $\kappa B$ Kinase Activation Mediated by the Linear Ubiquitin Chain Assembly Complex", Mol Cell Biol. 2014 Apr;34(7):1322-35. doi: 10.1128/MCB.01538-13

Buchini S, Gallat FX, Greig IR, Kim JH, Wakatsuki S, Chavas LM, Withers SG. “Tuning mechanismbased inactivators of neuraminidases: mechanistic and structural insights", Angew Chem Int Ed Engl. 2014 Mar 24;53(13):3382-6. doi: 10.1002/anie.201309675.

Suzuki T, Makyio H, Ando H, Komura N, Menjo M, Yamada Y, Imamura A, Ishida H, Wakatsuki S, Kato R, Kiso M. "Expanded potential of seleno-carbohydrates as a molecular tool for X-ray structural determination of a carbohydrate-protein complex with single/multi-wavelength anomalous dispersion phasing", Bioorg Med Chem. 2014 Apr 1;22(7):2090-101. doi: 10.1016/j.bmc.2014.02.023.

\section{Workshops}

S. Wakatsuki, "Structural basis of ubiquitin chain recognition and developments towards de novo phasing with XFEL", Structural Biology Department Seminar, Stanford School of Medicine, October 1, 2013

S. Wakatsuki "ID14: vision, concept and early days", invited talk at ESRF Users Meeting "Structural Biology at the ESRF: Past, Present \& Future”, ESRF, Grenoble, France, February 3, 2014

S. Wakatsuki, chair of workshop "Single Particle Imaging", SLAC, Menlo Park, California, USA, March 3-4, 2014

S. Wakatsuki, chair of session "Opportunities with new sources in structural biology" in American Crystallography Association, Albuquerque, New Mexico, USA, May 28, 2014

S. Wakatsuki, chair of session "Novel methods for structural life science research" in Annual meeting of Protein Society, Yokohama, Japan, June 25, 2014 
S. Wakatsuki, "New opportunities in structural biology with XFEL: from sources to structures", Keynote lecture at International Union of Crystallography Congress, Montreal, Canada, August 8, 2014

S. Wakatsuki, "Accelerator Based Structural Life Science", Japanese Association for Bioimaging, Osaka University, September 4, 2014

S. Wakatsuki, "SLAC Bioscience Strategy and Applications of XFEL to Structural Biology: the MFX Project and Two-color Mode", SLAC Photon Science Seminar, September 10, 2014

(อ)

\section{Questionnaire}

\section{Question}

Will follow-on funding (post-LDRD project) be applied for?

Source of support for follow-on funding?

Has follow-on funding been obtained?

Amount of follow-on funding $(\$ \mathrm{~K})$ ?

\section{Answer}

Y

DOE BER, NIH

Y (BER, NIGMS,

Stanford Hospital)

BER: $\$ 2,000,000$

NIGMS: $\$ 1,000,000$

Stanford Hospital:

$\$ 23,250$

0

Number of students supported by LDRD project?

0

Number of scientific staff/technical staff hired with LDRD funding? 1

Number of copyrights filed (beyond publications)?

0

Number of invention disclosures filed?

0

Number of patent applications filed? 


\subsection{An Automated Pipeline for Cosmological Mock Sky Survey Production}

Principal Investigator: Risa Wechsler, Co-Investigator: Tom Abel

\section{Project Description}

Galaxy surveys are rapidly becoming a leading probe of fundamental physics on the cosmic frontier, including dark energy, dark matter, inflation, and neutrino masses. However, cosmological inferences require precise predictions of structure formation and a detailed understanding of the relationship between galaxies and the matter distribution, for which cosmological simulations play an essential role. The work focuses on critical bottlenecks to efficient and accurate production of and access to simulated sky surveys: (1) production of multiscale initial conditions to improve the efficiency and scaling of cosmological codes; (2) integration of post-processing codes into various modular frameworks for more rapid analysis and development, (3) developing new techniques for simulating collisionless fluids by discretizing the dark matter sheet in phase space and (4) development of database solutions for efficient access of large simulated data sets.

\section{Accomplishments}

We have developed a pipeline for resimulating galaxy clusters at high resolution, selected from large cosmological boxes, including producing halo catalogs, merger and galaxy catalogs, using new algorithms designed for this project. This has been used to produce a suite of 100 cluster mass halos, and three papers have been published on the first scientific results from these simulations. More recently, this pipeline has been extended to simulate lower mass halos at higher resolution. We have made substantial improvements to a computational pipeline to go from cosmological parameters through simulations all the way to a full sky survey. This pipeline is being used and further developed to create sky surveys for the Dark Energy Survey, the Dark Energy Spectroscopic Instrument, and the Large Synoptic Survey Telescope. The first few full area full depth simulation from this pipeline has now been produced, which includes a realistic galaxy population as well as the impact of weak lensing. These simulations are being actively used by the full DES collaboration. We have now integrated these simulations into an image simulation pipeline developed by the ETH Zurich group, and this joint pipeline is being used to study systematics in the data. In addition, in collaboration with LSST scientists we have integrated these simulations with the LSST imaging pipeline, and are providing the simulations to the LSST Dark Energy Science collaboration. We have recently completed a 1 trillion particle simulation, the Dark Sky Simulation. Our analysis tools were used to process this data into halo catalogs. A new database server was set up to host and serve data from simulated sky surveys, this has been used to serve the dark sky data. This data was made publicly available just 10 weeks after the simulation completed running at Oakridge.

Abel, Hahn and Kaehler developed new visualization and analysis techniques relevant for cosmological dark matter simulations. The approach allows for new numerical methods to be 
developed that solve the dynamics of collisionless fluids discretizing by volume. This has led to important several papers, including one outlining new simulation techniques for dark matter, and one investigating implications for gravitational lensing. In addition, these techniques have allowed a new approach to simulating warm dark matter that solves a thirty-year-old problem in the field. Kaehler and Abel also began to investigate hybrid data formats that can outperform HDF5 formats, which are the current gold standard in our area.

\section{Publications}

O. Hahn \& T. Abel, “Multi-Scale Initial conditions for cosmological simulations". 2011, MNRAS, 415, 2101.

P. Behroozi, R. Wechsler, H. Wu. "The Rockstar Phase-Space Halo Finder". 2013, ApJ, 762, 31

P. Behroozi, R. Wechsler, "Gravitationally Consistent Halo Catalogs and Merger Trees for Precision Cosmology". 2013, ApJ, 763, 18

T. Abel, O. Hahn, R. Kaehler. "Tracing the Dark Matter Sheet in Phase Space". arXiv:1111.3944.

R. Kaehler, O. Hahn, T. Abel. A Novel Approach to Visualizing Dark Matter Simulations". arXiv:1208.3206.

O. Hahn, T. Abel, R. Kaehler.“ A New Approach to Simulating Collisionless Dark Matter Fluids". 2013, MNRAS, 434, 1171

H. Wu, O. Hahn, R. Wechsler, Y. Mao, P. Behroozi. Rhapsody: I. Structural Properties and Formation History from a Statistical Sample of Re-simulated Cluster-size Halos". 2013, ApJ, 763, 70

H. Wu, O. Hahn, R. Wechsler, P. Behroozi, Y. Mao. Rhapsody: II. Subhalo Properties and the Impact of Tidal Stripping from a Statistical Sample of Cluster-Size Halos". 2013, ApJ, 767, 23

R. Angulo, O. Hahn, T. Abel. , "The warm dark matter halo mass function below the cutoff scale", 2013, MNRAS, 434, 3337

R. Angulo, O. Hahn, T. Abel. , “How closely do baryons follow dark matter on large scales", 2013, MNRAS, 434, 3337

H. Wu, O. Hahn, A. Evrard, R. Wechsler, K. Dolag. Viral scaling of galaxies in clusters: bright to faint is cool to hot", 2013, MNRAS, 436, 460.

C. Chang, M. T. Busha, R. Wechsler, and others. 2013, MNRAS, 436, 460, "Modeling the Transfer Function for the Dark Energy Survey", 2014, arxiv:1411.0032 


\section{Questionnaire}

\begin{tabular}{ll}
\hline Question & Answer \\
\hline Will follow-on funding (post-LDRD project) be applied for? & Yes \\
\hline Source of support for follow-on funding? & DOE SciDAC \\
\hline Has follow-on funding been obtained? & Yes \\
\hline Amount of follow-on funding (\$K)? & 465 \\
\hline Number of Post Docs supported by LDRD project? & 2 \\
\hline Number of students supported by LDRD project? & 0 \\
\hline Number of scientific staff/technical staff hired with LDRD funding? & 1 \\
\hline Number of copyrights filed (beyond publications)? & 0 \\
\hline Number of invention disclosures filed? & 0 \\
\hline Number of patent applications filed? & 0
\end{tabular}

Notes on number of post-docs/students/staff: any post-doc or student who was supported for more than 10\% of his/her time should be included here. For staff, this is for staff that were hired specifically for and funded by the LDRD project (at least $50 \%$ of their time). 


\section{$20.0 \mathrm{cPix}^{2}$ : multi-gate area detector for $\mathrm{MHz}$ repetition rate pump- probe experiments}

Principal Investigator: Gabriella Carini

\section{Project Description}

Many scientifically interesting and technologically important investigations today, from understanding the mechanisms of charge separation in photosynthetic systems to the interaction of the electronic, atomic and magnetic lattices in multiferroics, are based on monitoring the response of a material, in time domain, after it is perturbed by an external stimulus. Signals in these experiments are often small requiring high repetition rates and photon counting for the needed statistic. But even slight beam motion or other instability can dominate the signal, making them nearly impossible. If, however, the probe signal is compared shot-by-shot with pump on and off, then the dominant sources of systematic error can be suppressed if not removed completely. Furthermore, low count rates, and an additional parameter (time) axis necessitates parallel detection scheme for realistic measurement times. Thus a 2D photon counter with at least two fast gates capable of operating at megahertz repetition rates is needed for the success of high repetition rate pump-probe experiments in SSRL's low alpha operation and at the proposed megahertz crab cavity beamlines in the Advanced Photon Source (APS) upgrade. No such detector, however, is currently available.

We have proposed to build a prototype pixel area detector with a $100 \times 100 \mu \mathrm{m}^{2}$ pixel size, designed for high repetition rate pump-probe experiments with at least two $100 \mathrm{~ns}$ gates, capable of at least $1.28 \mathrm{MHz}$ repetition rate, and 14-bit counting range.

A new prototype ASIC is being designed. The chip will be tested and bump-bonded at SLAC to a currently available pixelated sensor (Fig. 1a). A new PCB board will be designed and integrated in the currently available ePix camera assembly (Fig. 1b). The ePix DAQ and software interface will be modified accordingly. The prototype camera will be tested at SSRL. This validation with x-rays will serve as proof-of-concept for fast dynamic time domain studies needed to address some of DOE grand challenges. The showcase camera will be able to utilize high repetition rate and short bunches of existent and planned x-ray sources in US and around the world. This demonstrator would enable partnership with other laboratories and facilities. It will also provide SLAC with a technology enabling the development of fast counters for LCLS-II, useful for low photon density experiments with very sparse data. 

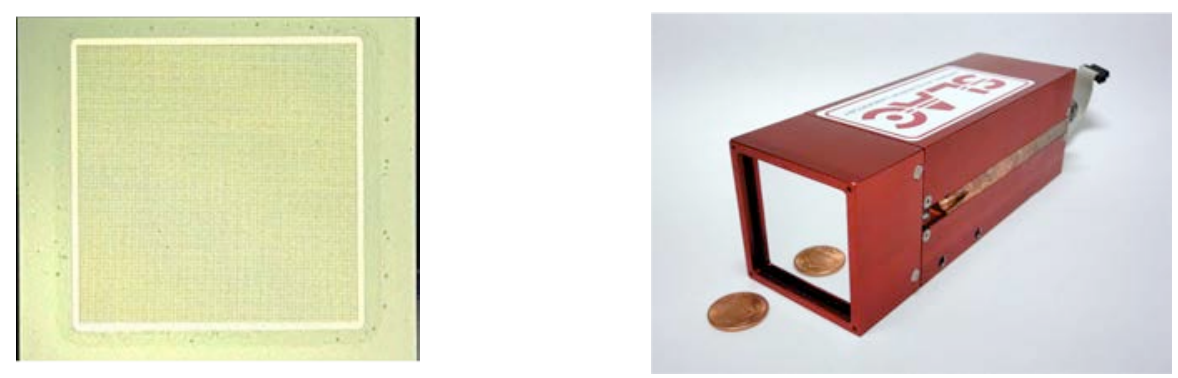

Fig. 1: a) ePix 10k sensor: designed and fabricated in house at SLAC and SNF. b) ePix-ONE camera assembly.

\section{Accomplishments}

During the first 6 months of the program we have focused the attention in developing the custom Application Specific Integrated Circuit (ASIC) which will be bump bonded to a silicon pixel array detector (PAD). In particular, the complete architecture has been finalized and validated through simulation to prove the required specifications.

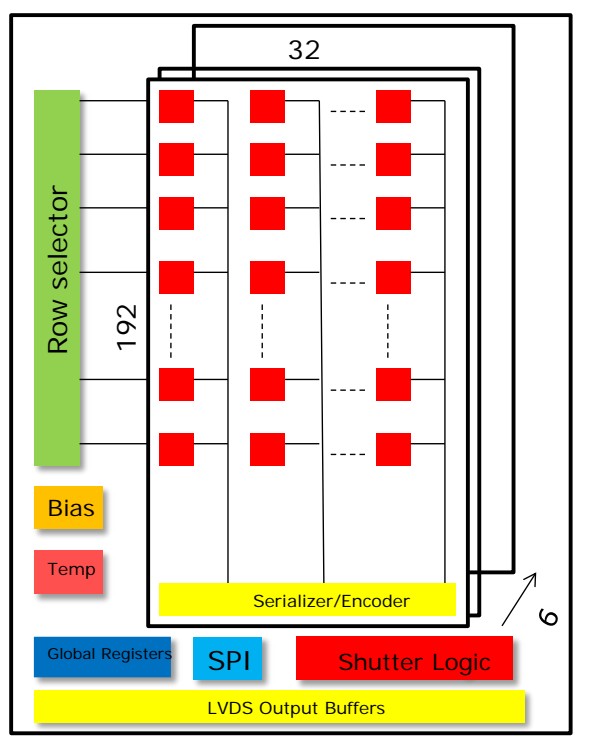

\begin{tabular}{|l|c}
\hline & Two Gate High Rep Rate Counter \\
\hline Processing time & $96 \mathrm{~ns}(76 \mathrm{~ns})^{\star}$ \\
\hline Counting rate & $1.28 \mathrm{MHz}(13 \mathrm{MHz})^{*}$ \\
\hline Counters per pixel & 2 (i.e. double gating) \\
\hline Counting length & 14 bits \\
\hline Thresholds & high $(15-32 \mathrm{keV})$ and low $(3-20 \mathrm{keV})$ \\
\hline Readout dead time & $<3 \mathrm{~ms}$ \\
\hline Pixel size & $\sim 100 \mathrm{um}$ \\
\hline Energy & $5 \mathrm{keV}-30 \mathrm{keV}$ \\
\hline Readout noise & $<350 \mathrm{e}-\mathrm{rms}$ \\
\hline Vacuum compatible & Yes \\
\hline Module & $\sim 150 \mathrm{kpixel}$ \\
\hline Operation mode & Synchronous \\
\hline
\end{tabular}

*specifications allow accommodating APS time structure.

Fig. 2: Left: ASIC architecture. b) Table summarizing the ASIC specifications.

Fig. 2 shows the architecture of the ASIC with the list of specifications. The ASIC is composed of three sections: an array of pixels that perform the counting operation, a row selector that addresses rows of the matrix for column parallel readout and a common section ("balcony") that is used to configure, control the phases of operation and readout the matrix. The design and simulations of the pixel circuitry have been completed together with part of the layout. Each pixel is composed of a charge preamplifier with continuous or time variant reset, a correlated double 
sampling baseline adjusting filter followed by a dual threshold discriminator and two counters (Fig. 3).

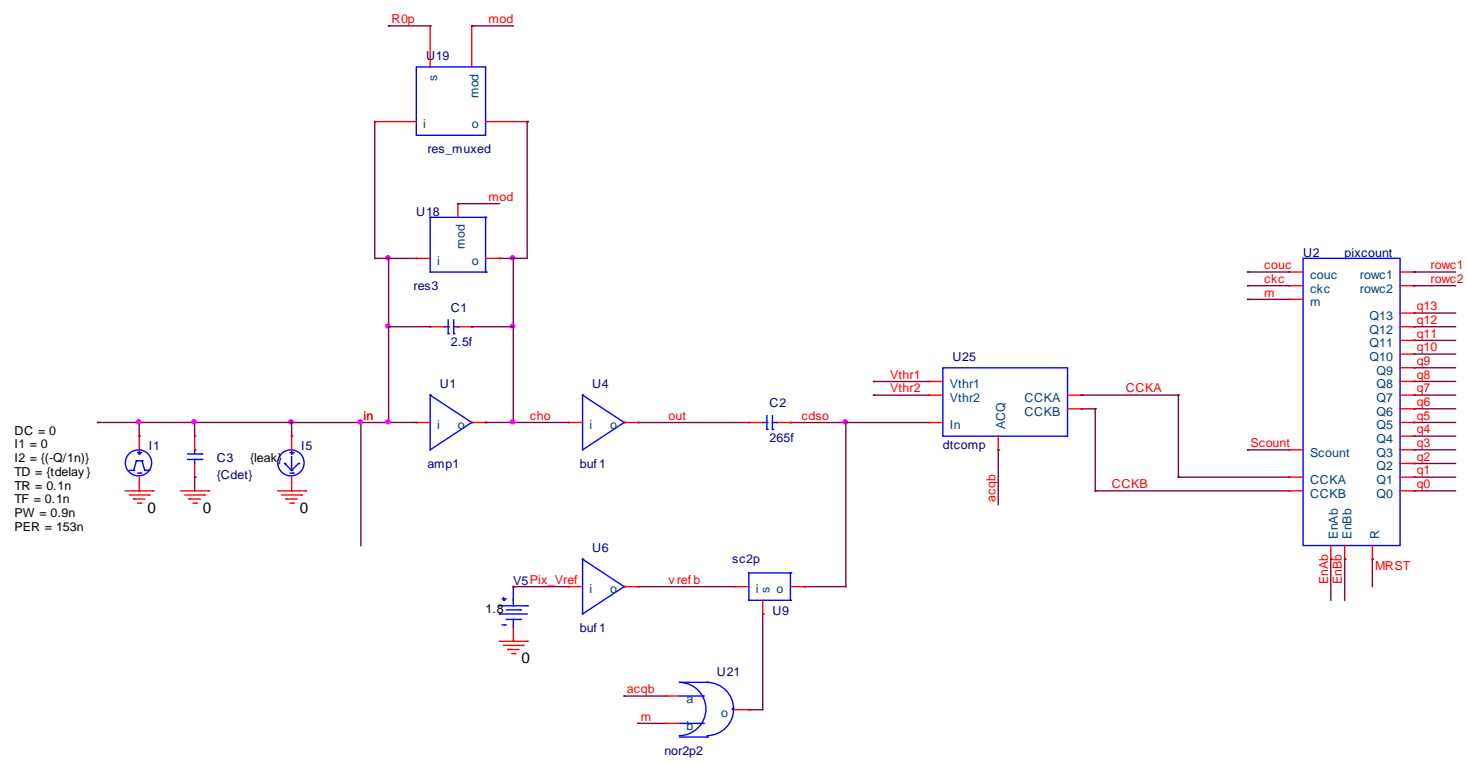

Fig. 3: Schematic of the pixel circuitry.

The circuit has been simulated at the maximum counting rate of $13 \mathrm{MHz}$ to be compatible with the characteristics of the APS most challenging time structure. Fig. 4 shows the results of such simulation.

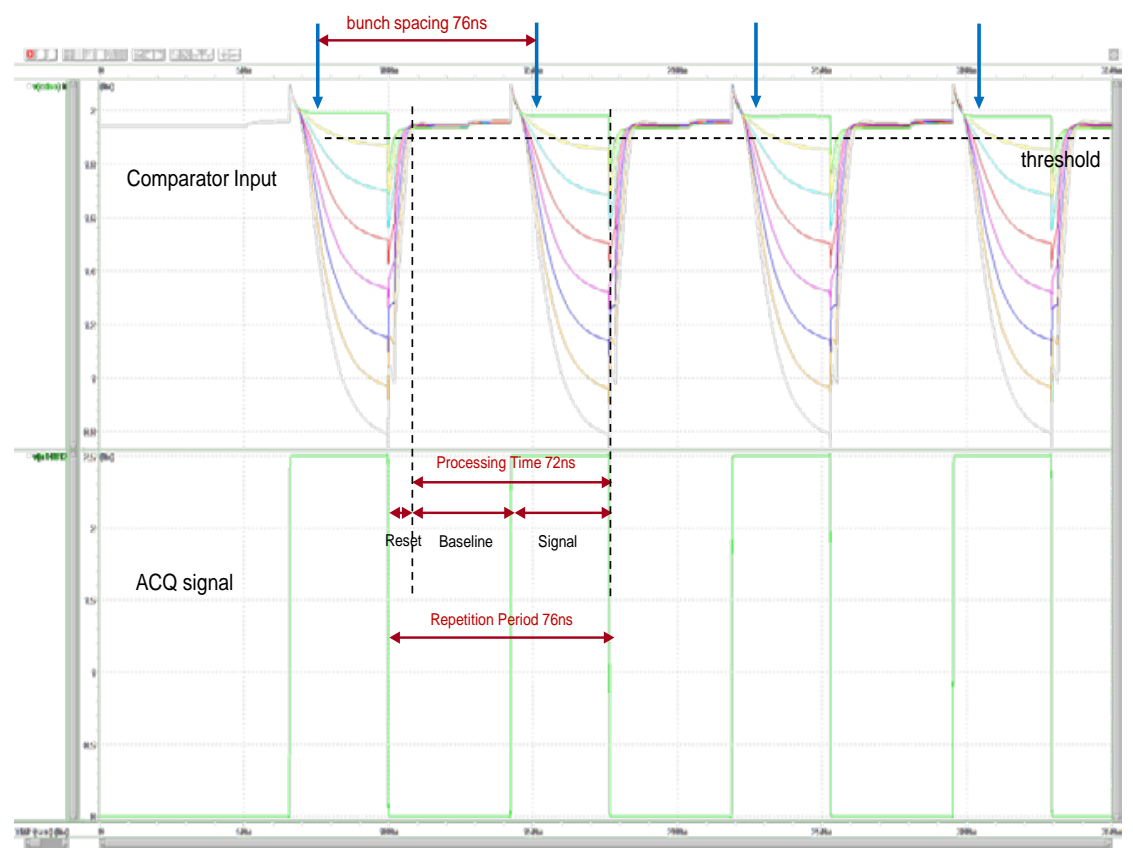

Fig. 4: Pixel circuitry simulated at the shortest repetition period of $76 \mathrm{~ns}$. 


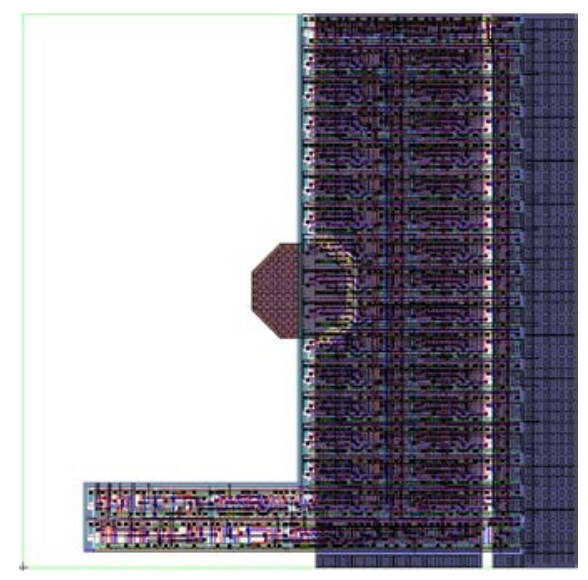

Fig. 5: Pixel layout.

The pixel circuit is now in the layout stage (Fig. 5). The row selection logic has been designed, simulated and layout. The "balcony" components have been designed and simulated. In particular, the simulations have demonstrated the capability of the high-speed serializers to sustain data rates up to $1 \mathrm{~Gb} / \mathrm{s}$. The serializer chain is composed of a $32 \times 1$ (or $64 \times 1$ ) 16 bit digital multiplexer followed by a buffer and a SLAC custom designed encoder based on the $8 \mathrm{~b} / 10 \mathrm{~b}$ protocol which has a define idle state and automatically packet each frame adding a start- and end-of-frame code to the data. The output of the encoder, named SSP (Simple Streaming Protocol) Framer, feeds into a serializer and a LVDS transmitter. Fig. 6 shows a simulation for a worst case of $664 \times 1$ channels running at a data rate of $1 \mathrm{~Gb} / \mathrm{s}$.

6 LVDS digital outputs each one multiplexing 64 columns

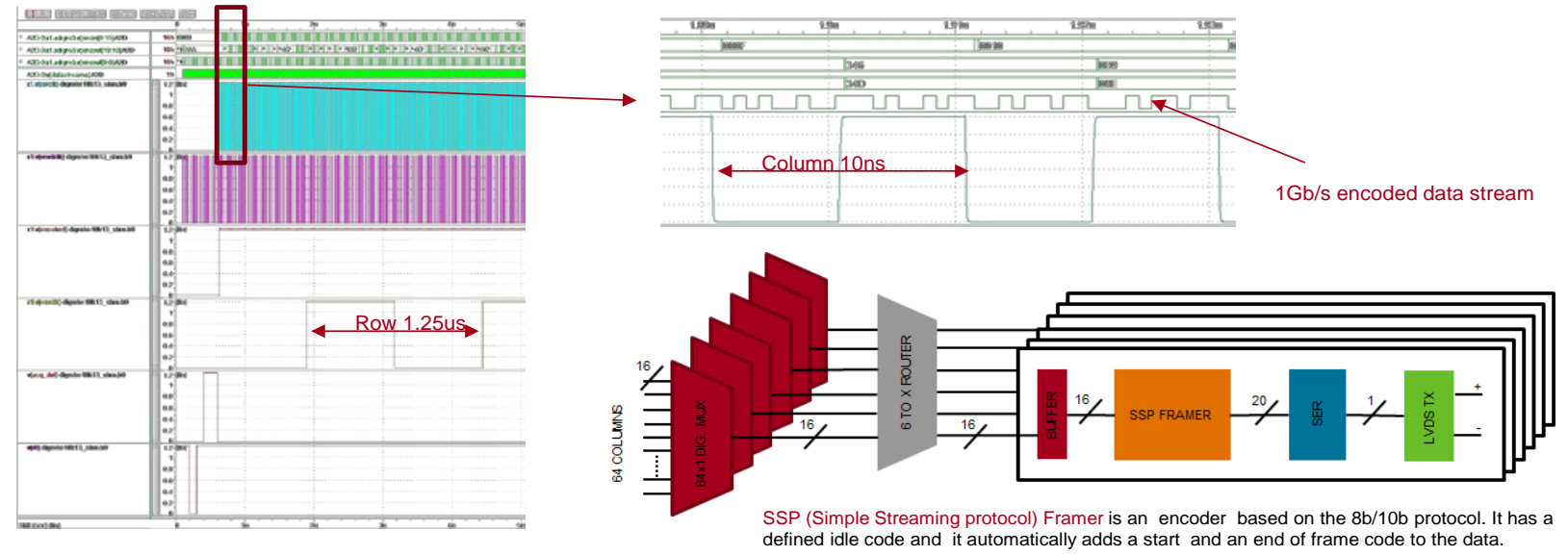

Fig. 6: Simulations demonstrating the data rate capability of the high-speed serializers.

The balcony is also in layout stage. The layout phase is now in the completion phase in line with the scheduled plan that considers a submission for fabrication toward the end of December 2014. 
In addition to provide SLAC with a technology that can be the foundation for the development of fast counters for LCLS-II, there are other experiments that could benefit from this R\&D effort. For example, one technique that can be addressed is dispersive spectroscopy using crystal arrays, where a photon's energy is determined by recording its angle using a spatially segmented detector. Such methods can achieve energy resolutions well below an eV and are critical tools for measuring chemical shifts of x-ray emission spectra. A better understanding of atomic scale catalysis (including artificial photosynthesis) is crucial in discovery and deployment of next generation of catalysts needed to solve many of our energy challenges. But the atomic scale changes in catalysis are subtle and the transition states are short lived. Ability to monitor and measure these changes and catch the short lived transition states are the critical scientific drivers for chemical dynamics investigations at SSRL and one of the prime motivation behind LCLS-II.

A spectrometer with high energy resolution, simultaneously with large solid angle coverage (and hence throughput) with ability to normalize the spectral signal at a $\mathrm{MHz}$ - and thus extract very small changes is essential for these experiments to succeed.

A spectrometer built on appropriate crystal optics and a cPix ${ }^{2}$ detector can fulfill these needs.

We have available another sensor which would allow a small prototype circuit chip with a twodimensional pixel geometry to readout a one dimensional array of strips as shown in Fig. 7. In this design 384 out of the total 2034 pixel channels are employed to read out two rows of 192 strips each. Individual strips are 100 by 500 ent. This allows a circuit chip with a modest width (i.e. 5 $\mathrm{mm}$ ) to span a much larger angular span corresponding to $20 \mathrm{~mm}$.

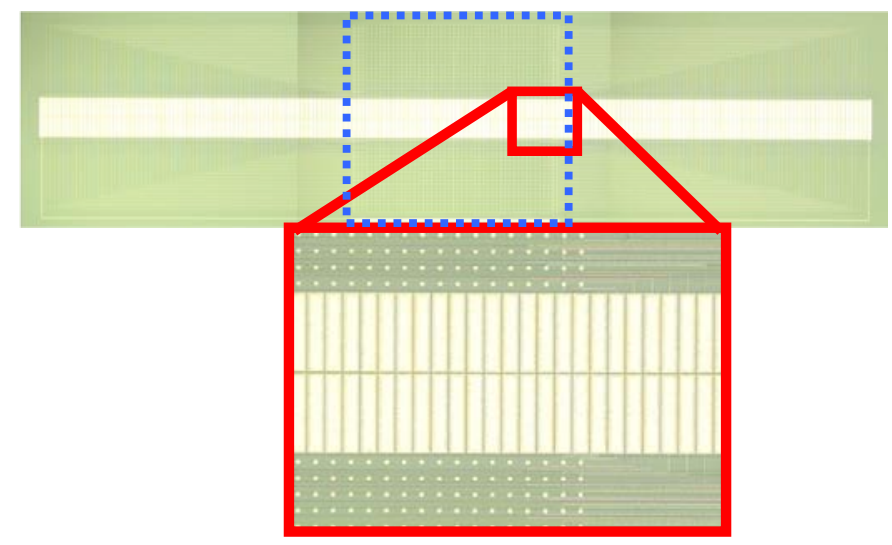

Fig. 7: Sensor designed for dispersive spectroscopy with a fan-out from the first-generation, pixelated, circuit chip to a linear array of two by 192 short strips (top); each strip element is 100 by $500 \square \mathrm{m}^{2}$. Close up image of strip elements connected to bump-bond pads, which allow this long-strip array to be readout be a small two-dimensional pixel circuit (indicated by blue dashed square). These sensors were fabricated at the Stanford Nanofabrication Facility.

\section{Publications}

C. J. Kenney et al. "Photon-Counting Detectors for Pump-Probe Science", IEEE-NSS Conference Records, Seoul, Korea (2013). 


\section{Workshops}

None

\section{Questionnaire}

Question Answer

Will follow-on funding (post-LDRD project) be applied for?

Source of support for follow-on funding?

Has follow-on funding been obtained?

Amount of follow-on funding $(\$ \mathrm{~K})$ ?

Number of Post Docs supported by LDRD project?

Number of students supported by LDRD project?

Number of scientific staff/technical staff hired with LDRD funding?

Number of copyrights filed (beyond publications)?

Number of invention disclosures filed?

Number of patent applications filed?
$\mathrm{N} / \mathrm{A}$

N/A

N/A

N/A

N/A

N/A

N/A

N/A

None

None 